\section{Conferences, Schools and Programmes}

In 1992, Europhysics News publishes two Meetings Issues: in March and November. Urgent up-datings can also be included in all issues in the form of advertisements charged at SFR 100.- per insertion.

The events listed here are those for which details were received at the EPS Secretariat before 30 October 1992. In general, al events interesting European physicists which take place from the beginning of 1993 are cited. For events prior to that date readers are referred to the March 1992 issue.

The meetings are in date order divided into three categories - Conferences, Schools and Programmes, although the distinction between these three categories is not always evident. Europhysics Conferences, i.e. meetings organized or co-organized by EPS, its
Divisions and Sections, are printed entirely in bold type and are boxed. Events sponsored by EPS have all information printed in bold type; Europhysics Study Conferences have an $\mathbf{E}$ preceding the date and are also boxed; European Research Conferences in Physics organized in cooperation with the ESF, Strasbourg, are boxed.

For inclusion in the March 1993 issue organizers should send details to the EPS Secretariat by 25 February 1993 at the latest. Even if full details are not yet available, the preliminary information should be given.

Entries are selected from a database compiled using information which is supplied by organizers and by meetings correspondents.

Application for sponsorship of a meeting should be made to the EPS Secretariat.

\section{Conferences 1993}

\section{JANUARY}

Jan. 2-11 Statistical Description of Transport in Plasma, Astro- and Nuclear Physics: Interdisciplinary Workshop Les Houches, France J. Misguich, Assocn. EURATOM-CEA, Centre d'Etudes de Cadarache, F-13108 Saint-Paul-les-Durance Cédex + 33 () $42254667 / 42256233 \quad$ stpf @ tucana.cad.cea.fr $\quad$ Ab: 15 Oct 92 / PP / $<70$ FF 4700.-; incl. board, lodge; incl. proc.

3-7 American Astro. Soc. 181th Meeting Phoenix AZ, USA D. Burstein, Arizona State Univ., Phoenix AZ, USA

4-7 High- $T_{c}$ Superconductivity: Conf. Eilat, Israel S. Cahana, Min. of Sci. \& Tech,, POB 18195 IL-91811 Jerusalem

4 - March 19 Gordon Res. Confs.

4- 8 Jan. Polymers: Superconductivity

11- 15 Jan. Composites

18-22 Jan. Electrochemistry

1- 5 March Structures, Energetics \& Reaction Dyn of Gaseous lons

8-12 March Chemical Reactions at Surfaces

15-19 March Crystal Growth: STM Ventura, CA USA A.M. Cruickshank, Gordon Res. Center, Univ. of Rhode Island, Kingston, RI 02881-0801, USA +1 (401) $7834011 / 7837644$ bcp101 @ uriacc 5 - 9 Intermediate \& High-Energy Nuclear Physics: 10th Nordic Meeting Gräftavallen, Sweden L. Westerberg, Dept. of Radiation Sciences, The Svedberg Lab. Uppsala Univ, POB 535, S-751 21 Uppsala + 46 (18) $183060 / 183833$ westerberg @ tsl.uu.se $\approx$ SKR 3100.-; incl. board, lodge 10-16 Computational Methods for Nonlinea Phenomena Oberwolfach, Germany Math. Forschungsinstitut, Albertstrasse 24, W-7800 Freiburg $-/+49$ (761) 272698

11- 13 Computational Cond. Matter Physics: 6 th Int. Workshop Trieste, Italy ICTP, POB 586, 1-34100 Trieste +39(40)22401/224163 11-14 Spectroscopic Ellipsometry: 1st Int. Conf. (ICSE '93) Paris, France Boite 82, Univ. P. \& M. Curie, 4, pl. Jussieu, F-75252 Paris Cédex 05 $-1+33$ (1) 44273982

11 - 14 Cataclysmic Variables \& Related Phys ics: 2nd Technion-Haifa Conf. Eilat, Israe O. Regev, Physics Dept., Technion, Haifa 32000 , Israel $-/+962$ (4) 221514

11-15 Very High Angular Resolution Imaging IAU Symp. No. 158 Sydney, Australia J. Davis, Chatterton Astronom. Dept, School of Physics, Univ. of Sydney, Sydney, NSW 2006, Australia SUS 135.11 - 15 Particle Acceleration Phenomena in Astrophysical Plasmas: IAU. Coll. No. 142 College Park, ML, USA E. L. Chupp, Physics Dept., Univ. of New Hampshire, Durham, NH 03824, USA - $/+1$ (603) 8622998
11- 29 High-Temp. Superconductors \& Related Materials (Adv. Activities): Exp. Workshop Trieste, Italy ICTP, POB 586, I-34100 Trieste +39(40) $22401 / 224163 \quad$ A: 15 Aug $92 / \mathrm{lim}$

18-22 UNK B-Factory Workshop Liblice Castle, Czecho-Slovakia F. Ferroni vaxrom::ferron 18 - 23 Gross Props. of Nuclei and Nuclear Excitations: 21st Int. Workshop Hirschegg, Austria H. Feldmeier, GSI, Postfach 1105 52, W-6100 Darmstadt $11+49(6151) 359744 /$ - tp36 @ ddagsi3 $20-23$ Neutrons \& X-Rays in the Study of Magnetism: Int. Workshop Grenoble, France D. Dijoux, SCARPO, ILL, BP 156, F-38042 Grenoble Cédex 9 $-/+33$ () 76483906 blank @ frill51 Ab: 1 Nov $92 / \mathrm{PP}$

25 Ballistic Electron Microscopy \& Related STM-Based Techniques: Workshop Williamsburg, VA, USA R. Ludeke, IBM Res. Div., Yorktown Heights, NY 10598, USA $\quad-1+1$ (914) 9452536 25-27 Unified Symmetry in the Small \& in the Large: Conf. Coral Gables, FL, USA Global Fund, Inc., 1450 Madruga Ave., Suite 301, Coral Gables, FL 33146-3164, USA +1 (305) 6699411 6699464

25-29 Physics \& Chemistry of Semiconductor Interfaces: Conf. Williamsburg, VA, USA L.J. Brillson, Xerox Webster Res. Center, 800 Phillips Rd. 0114-41 D, Webster, NY 14580, USA

25-29 Nuclear Physics: 31st Int. Winter Meeting Bormio, Italy I. Iori, Dip. di Fisica, via Celeria, 16 I-20133 Milano bormio (1) vaxmi.infn.it

\section{6-30 Kinetic Processes in Minerals \&} Ceramics: European Res. Conf. in Physics Castelvecchio, nr. Pisa, Italy J. Hendekovic ERC, ESF, 1, quai Lezay Marnésia, F-67000 Srasbourg Cédex +33 () $88767135 / 88366987$ A: 31 Oct 92 / Ab: 31 Oct 92 / NP/ $100 /$ FF 4000.incl. board, lodge ESF, CEC

31 - Feb. 5 Electronic Imaging - Sci. \& Techn. Symp. San Jose, CA, USA SPIE, POB 10 Bellingham, WA 98227-0010, USA

$6763290 / 6761445$

+1 (206)

PP: 4 Jan 93 / SUS 420

30 - Feb. 6 Perspectives in Neutrinos, Atomic Physics \& Gravitation: XXVIII Rencontres de Moriond Villars, Switzerland J. Trân Thanh Vân, LPTHE, Bât. 211, Univ. de Paris-Sud, F-91405 Orsay Cédex +33 (1) $69285135 / 69288659$ PP 130 / inv.; incl. board, lodge; incl. proc. CEC, CNRS, 30 - Feb. 6 Nuclear Dynamics: 9th Winter Work$\begin{array}{lll}\text { shop Key West, FL, USA } & \text { B. Back, ANL, }\end{array}$ Argonne, IL 60439, USA +1 (708) 2523618 / 2526210 back (1) anlphy

31 - Feb. 6 Asymptotics \& Adaptivity in Computational Mechanics Oberwolfach, Germany Math. Forschungsinstitut, Albertstr. 24, W-7800 Freiburg $\quad-/+49(761) 272698$

\section{Key to Abbreviations}

A: Application deadline

$\mathrm{Ab}$ : Abstracts deadline

inv.: restricted to invited applicants

NP: no proceedings will be published

$P$ : Papers deadline (information on proceedings has not been received)

PP: proceedings will be published (papers deadline)

$x: \quad$ no. of participants $(<x$ : restricted to $x)$

lim.: limited participation

+: country (area) tel. / fax

email - bitnet address or other

The information is given in this order:

Date Title

Venue

Contact for information

A: ... / Ab: ... / NP, PP or P: Papers dead line / Number of participants / inv. / languages other than English / Conference fee Sponsor

$>$ after the title indicates an important amendment to the last published entry.

\section{Meetings Listings}

The Europhysics Meetings Database carries a complete listing of physics meetings worldwide of interest to European physicists. Listings sorted by date, venue and field can be ordered from the EPS Secretariat, Geneva (SFR 50.- per 50 entries).

\section{Meetings: 502 (1993); 96 (1994)}

Listed in this issue: $569(1993 / 4)$

\section{FEBRUARY}

Feb. Turbulence in Space Plasmas: Workshop Aussois, France M. Lefeuvre, Ave. de la Recherche Scientifique 3A, F-45071 Orléans Cédex IURS Feb. Optical Fibres \& Their Applicns. Warsaw, Poland R.S. Romaniuk, IPE, Warsaw Univ. of Tech. Nowowiejska 15-19, PL-00-665 Warsaw +48 (22) $253709 / 252300$

1-3 Superconductivity: 5 th Annual Conf. (SC Global 92) San Jose, CA, USA Superconductor Applcns. Assocn., 27692 Deputy Circle, Laguna Hill, CA 92653, USA

1-4 Infrared Solar Physics Tucson, AZ, USA D. Rabin, Nat. Solar Obs., POB 26732, Tucson, AZ 85726, USA

4-6 From Weak Neutral Currents to the W/Z \& Beyond: Int. Symp. Los Angeles, CA, USA M. Laraneta, Physics Dept., UCLA, 405 Hilgard Ave. Los Angeles, CA 90024-1547, USA +1 (213) $8254649 / 2061091 \quad$ laraneta (d) uclahep

4-8 Intense Lie Groups \& Their Physical Applens.: NATO ARW San Antonio, TX, USA R. Wilson, Div, of Math, Univ. of Texas, San Antonio, TX 789249, USA

10 Silicon-Based Optical Electronics London, UK $\quad$ J. McDonald, IEE Conf. Services, Savoy Place, London WC2R OBL, UK +44 (71) $2401871 / 4973633 \quad$ IOP

14- 18 Biophysical Soc. 37th Ann. Meeting Washington, DC, USA E.M. Gray, Biophysica Soc., 9650 Rockville Pike, Bethesda, MD 20814, USA 14 - 18 Magnetohydrodynamics - Flows \& Turbulence: 7 th Beer-Sheva Int. Seminar Jerusalem, Israel H. Branover, Center for MHD Studies, BenGurion Univ., POB 653, Beer-Sheva, Israel $\quad-/+972$ (57) 280467

15-17 Nonlinear Phenomena in Complex Systems Polatsk, Belarus V.I. Kuvshinov, Inst. of Physics, Acad. of Sciences, F. Scaryna Av. 70 CIS-220072 Minsk +7(0172) $394559 / 393131$ kilin @ adonis.ias.mk.su A: 15 Dec $92 /$ Ab: 15 Dec 92 / PP: 15 Feb 93 
21-26 Optical Fiber Communications Conf. (OFC '93) San Jose, CA, USA OSA, Meetings Dept., 2010 Massachusetts Ave. NW, Washington, DC 20036, USA +1(202) $2238130 / 2231096$ 22 - 26 Monte Carlo Simulation: Int. Conf. Tallahassee, FL, USA Supercomputer Computations Res. Inst., FSU, Tallahassee, FL 32306, USA mc93@ scri.fsu.edn

\section{$\mathrm{MARCH}$}

Spring Nonlinear Dynamics of Single Particles: Adv. ICFA Beam Dynamics Workshop A. Hofmann CERN, $\mathrm{CH}-1211$ Geneva 23 +41(22) 7676972 I $7830552 \quad$ albert @ cernvm.cern.ch

March Optical Props. of Low-Dimensional Silicon Structures: NATO ARW Meylan, France D. Benshal, CNET-CNRS, France Telecom, BP 98 F-38243 Meylan Cédex

1-3 Ergodic Concepts in Stellar Dynamics Workshop Geneva, Switzerland D. Pfenniger, Geneva Obs., 51, ch. des Maillettes, $\mathrm{CH}-1290$ Sauverny $+41(22) 7552611 / 7553983$ pfenniger (a) cgeuge54 A: 15 Dec $92 / 40$

1 - 4 Static \& Dynamic Light Scattering - Techniques \& Applicns. to Dense Colloids: Meeting (SDLS-2)

Burg on Fehmarn, Germany

K. Schaetzel, Inst. f. Angewandte Physik, Leibnitzstr. $11, \mathrm{~W}-2300 \mathrm{Kiel}$

A: 31 Dec 92 / Ab: 30 Nov 92

2 - 4 High-Energy Solar Phenomena - A New Era of Spacecraft Measurement: Conf. Waterville, $\mathrm{NH}$, USA J. Ryan, Univ. of New Hampshire, Durham, $\mathrm{NH} 03824$, USA

2-5 Quantum Interferometry: Adriatico Res. Conf. Trieste, Italy ICTP, POB 586, I-34100 Trieste $+39(40) 22401 / 224163$

3- 4 Semiconductor Growth, Surfaces \& Interfaces: Disc. Meeting London, UK Royal Soc. of Chem., Burlington House, London W1V OBN, UK +44 (71) $4378656 / 7341227 \quad$ PP / $<300$

5-8 Microscopy of Semiconducting Materials: 8th Oxford Conf. (SMM VIII) Oxford, UK RMS $37 / 38$ St. Clements, Oxford OX4 1AJ, UK +44(865) $248768 / 791237$ Ab: 1 Dec 92 IoP, MRS 7-12 Liquid Crystals: European Conf. (ECLC93) Flims, Switzerland M. Schadt F. Hoffmann-La Roche, Dept. RCLR, F. HoffmannLa Roche Ltd., CH-4002 Basel +41 (61) 6884584 / $6881466 \quad$ Ab: 1 Oct 92 / NP / 250 SFR 300.- (400.- after $1 \mathrm{Dec} 92)$; incl. proc.

8-10 German Soc. of Rheology Ann. Meeting Karlsruhe, Germany K.-H. Habig, DRG, Unter-der Eihen 87, W-1000 Berlin 45

8-12 Highlights of Particle \& Cond. Matter Physics: Conf. (SALAMFEST) Trieste, Italy ICTP POB 586, I-34100 Trieste $\quad+39(40) 22401$ 224163

8-12 Frühjahrstagung der DPG: Extraterrestrische Physik, Kurzzeitphysik, Plasmaphysik

Greifswald, Germany German Phys. Soc. Haupstr. 5, W-5340 Bad Honnef 1 +49 (2224) 710 61/710 63 Ab: 1 Dec 92

9-11 Electromagnetic Compatibility: 10 th Int. Symp. Zurich, Switzerland G. Meyer, ETH Zentrum-IKT, CH-8092 Zurich +41 (1) $2562790 /$ 2620943

10-12 Deutschen Ges. für Kristallographie 31 Jahrestagung Bochum, Germany Mrs. Schwerte Inst. f. Mineralogie, Univ. Bochum, Postfach 102148 , W-4630 Bochum 1 DPG

13- 20 Electroweak Interactions \& Unified Theories: XXVIII Rencontres de Moriond Les Arcs, France J. Trân Thanh Vân, LPTHE, Bât. 211 Univ. de Paris-Sud, F-91405 Orsay Cédex +33 (1) $69285135 / 69288659 \quad$ A: 15 Jan 93 / PP 130 / inv. / FF 4970.-; incl. board, lodge; incl. proc. CEC, CNRS, CEA, NSF

13- 20 The Cold Universe: XIII Moriond Astrophysics Meeting Les Arcs, France J. Trân Thanh Vân LPTHE, Bât. 211, Univ. de Paris-Sud, F-91405 Orsay Cédex +33 (1) $69285135 / 69288659$ A: $15 \mathrm{Jan}$ 93 / PP / 130 / inv. / FF 4970.-; incl. board, lodge; incl. proc. CEC, CNRS, CEA, NSF

13-20 33rd Sanibel Symp. St. Augustine, FL, USA Univ. of Florida, Gainesville, FL 32611 2085 , USA +1 (904) $3921597 / 3928722$ sanibel @ qtp.ufl.edu
15- 18 Theorien jenseits des Standardsmodells Bad Honnef, Germany German Phys. Soc., Haupstr. 5, W-5340 Bad Honnef 1 +49 (2224) 71061 / 71063

15 - 19 Lunar \& Planetary Sci. Conf. Houston, TX, USA P. Jones, Program Services Dept., LPI, 3600 Bay Area Blvd., Houston, TX 77058, USA

15-19 Frühjahrstagung der DPG: Atomphysik, Massenspektrometrie, Molekülphysik, Quantenoptik Berlin, Germany German Phys, Soc., Haupstr. 5 , W-5340 Bad Honnef 1

71063 Ab: 1 Dec 92

15-April 2 Representation Theory of Lie Groups: Workshop Trieste, Italy ICTP, POB 586 1-34100 Trieste +39(40) $22401 / 224163$

17 - 19 Tagungen der DPG: Didaktik Esslingen, Germany K. Luchner, Sektion Physik de Univ. Schellingstr. 4, W-8000 Munich 40

18-20 Finnish Phys. Soc. 27th Ann. Meeting Turku, Finland S. Nokkanen, Siltavuorenpenger 20M, SF-00170 Helsinki +358 (0) 1918375 1918378 nokkanen @ phcu.helsinki.fi A: 31 Jan 93 / Ab: 31 Jan 93 / PP / 400, Finnish / FIM 900.90.- students; incl. proc.

20-27 QCD \& High-Energy Hadronic Interactions: XXVIII Rencontres de Moriond Les Arcs, France J. Trân Thanh Vân, LPTHE, Bât. 211, Univ. de Paris-Sud, F-91405 Orsay Cédex +33 (1) 69285135 / 69288659 A: 15 Jan 93 / PP / $130 /$ inv. / FF 4970. - ; incl. board, lodge; incl. proc. CEC, CNRS, CEA, NSF

22 - 24 Dynamics of Disordered Materials: 2nd Int. Workshop Grenoble, France W. Petry, ILL. BP 156, F-38042 Grenoble Cédex $9+33$ () $76207187 / 76483906$ petry @ frill51 /PP 22 - 24 Robotic Observatories - The 1st Decade: Conf. Tucson, AZ, USA B. Rafert, Dept. of Physics \& Space Sci., Florida Inst. of Tech., $150 \mathrm{~W}$. Univ. Blvd., Melbourne, FL 32901, USA

22-26 American Phys. Soc. March Meeting Seattle, WA, USA APS, 335 E. 45 th St., New York, NY 10017, USA $\quad+1$ (212) $6827341 / 6872532$ A: 26 Feb 93 / PP: 4 Dec $92 /$ SUS 290.

22-26 Theory of Accretion Disks: NATO ARW Garching, Germany F. Meyer, Inst. f. Astrophysik, Karl-Schwarzschild-Str. 1, W-8046 Garching

22- 26 57. Physikertagung der DPG + Fachgremien: Geschichte der Physik, Gravitation und Relativitätstheorie, Physik der Hadronen und Kerne, Strahlenwirkung und-schutz, Teilchenphysik, Theoretische und Mathematische Grundlagen der Physik, sowie dem Arbeitskreis Energie und dem Beratenden Ausschuss der Industriephysiker Mainz, Germany German Phys. Soc., Haupstr. 5, W-5340 Bad Honnef 1 +49 (2224) $71061 / 71063$ Ab: 1 Dec 92

24-26 Swiss Phys. Soc. Spring Meeting Neuchâtel, Switzerland W. Amrein, Sect. de Physique, Univ. de Genève, 24, quai E.-Ansermet, $\mathrm{CH}-1211$ Geneva 4 +41 (22) $7026310 / 7812192$. Ab: 10 Jan 93 / PP / 200 / German, French

28-April 1 Mechanisms in Radiation Biology CEC Workshop Bad Honnef, Germany German Phys. Soc., Haupstr. 5, W-5340 Bad Honnef $1+49$ (2224) $71061 / 71063$

29-31 Spatio-Temporal Props. of Centrifugal Instabilities: NATO ARW Nice, France P. Laure Inst. Nonlinéaire, UMR CNRS 129, Univ. of Nice, F-06108 Nice

29 - April 1 Phys. Soc. of Japan 48th Ann. Meeting $>\quad$ Sendai-shi, Japan D.Y. Takada, PSJ, Kikai-Shinko Building, 3-5-8 Shiba-Koen, Minato-ku, Tokyo 105, Japan +81 (3) $34342671 / 34320997$ 29 - April 1 Japan Soc. of Applied Physics Spring Meeting Tokyo, Japan JSAP, Kunimatsu Bldg. 1-2-6 Kudan-kita, Chiyoda-ku, Tokyo 102, Japan +81 (3) $3281041 / 2216245$

29-April 1 DAGA: 19. Tagung Frankfurt, Ger many A. Lacroix, Inst. f. Angewandte Physik, Univ. Frankfurt, Postfach 1119 32, W-6000 Frankfurt-amMain $11 \quad-/+49(69) 7988383 \quad$ Ab: 1 Dec $93 /$ DM 250.-

29- April 1 Acoustical Sensing \& Imaging: Int. Conf. (ASI93) London, UK IEE Conf. Services, Savoy Place, London WC2R OBL, UK $\quad+44(71)$ $2401871 / 4973633$ Ab: 17 Aug 92

29-April 2 Nuclear Physics: 2nd Biennial Workshop Megève, France J.P. Burq, Inst. Physique Nucléaire, Univ. Claude Bernard-ISM, 43, blvd. du 11 novembre 1918, F-69622 Villeurbanne Cédex +33 () $72431062 / 72448004$
29-April 2 European Phys. Soc. Cond. Matter Div. 13th Gen. Conf. + Arbeitskreis Festkörperphysik bei der DPG Regensburg, Germany H. Hoffmann, Inst. f. Angewandte Physik, Univ. Regensburg, W-8400 Regensburg +49(941) $9432621 / 9434544$ A: 1 Dec 92 / Ab: 1 Dec 92 / PP / 3000 / DM 350.-; 200.- IOM's; 150.- students; 90.- IOM students

30-April 1 Nuclear \& Particle Physics Conf. Glasgow, UK I.J.D. MacGregor, Kelvin Lab. Glasgow Univ. dmacc (9) uk.ac.gla.ekva IoP 31 - April 1 Ultraviolet Radiation Sources, Exposures \& Effects York, UK D. Crawford, Physics Dept., Royal Marsden Hospital, Sutton SM2 5PT, UK - I+44 (81) $6433812 \quad$ IOP

\section{APRIL}

April 4-8 European Union of Geosci.: Biennial Meeting (EUG VII) Strasbourg, France Geological Survey of The Netherlands, Postbus 157 NL-2000 AD Haarlem $\quad-/+31$ (23) 351614 Ab: 1 Dec 92

5-7 Analysis by a Combination of lon-Beam (Accelerator-Based) \& Surface Specific Techniques: 3rd Int. Conf. on Ion-Beam \& Surface Specific Analysis Techniques (IB-SA/93) Namur, Belgium G. Demortier, LARN, Fac. Universitaires, N.-D. de la Paix, 22, rue Muzet +32 (81) $731267 / 737938$ A: 1 Mar 93 / Ab: 1 Mar 93/ NP / BF 4000 .

5-7 Sci. \& Technology in Third-World Development Glasgow, UK R. Heeks, IDPM, Manchester Univ., Precinct Centre, Manchester M13 9QS, UK

5-8 Atomic, Molecular \& Optical Physics: 3rd Nat. Conf. Chester, UK D.M.P. Holland, Daresbury Lab., Daresbury, Cheshire WA4 4AD, UK

6 The Netherlands' Phys. Soc. Spring Meeting Utrecht, The Netherlands A.M. Hoogenboom, NNV, Julianalaan 74, NL-3722 GS Bilthoven +31 (30) $285626 / 251381$

6-8 Numerical Analysis of Semiconductor Devices \& Integrated Circuits: 9th Int. Conf (NASECODE IX) Copper Mountain, CO, USA

P. McKeever, 26 Temple Lane, Dublin 2, Ireland

+353 (1) $6797655 / 6792469$ jmiller @ vax1.tcdie 12- 15 American Phys. Soc. \& American As socn. of Physics Teachers: Joint Spring Meeting Washington, DC, USA APS, 335 E. 45th St., New York, NY 10017, USA $\quad+1$ (212) $6827341 / 6872532$ 12-16 Materials Res. Soc. Spring Meeting San Francisco, CA, USA M.N. Geil, MRS, 9800 Mc Knight Rd., Pittsburg, PA 15237, USA +1 (412) $3673003 / 3674373$

12-16 Optical Engng. \& Photonics in Aerospace Sci. \& Sensing: SPIE Int. Symp. (OE/Aerospace Sensing '93) Orlando, FL, USA SPIE, POB 10 Bellingham, WA 98227-0010, USA

$6763290 / 6761445$

13-16 Magnetics: Int. Conf. (INTERMAG) Stockholm, Sweden Congrex, POB 5619, S-11486 Stockholm $\quad-I+46(8) 6126292$

13-17 Spatio-Temporal Patterns in Nonequilibrium Complex Systems: NATO ARW Santa Fe, NM, USA P.E. Cladis, AT\&T Bell Labs, 600 Mountain Ave., MH1E441, Murray Hill, NJ 07974, USA

13-23 Phase Transitions \& Relaxation in Systems with Competing Energy Scales: NATO ASI Geilo, Norway G. Jarret, Physics Dept., Inst. for energiteknik, POB 40, N-2007 Kjeller +47 (6) $806075 / 810820$ gerd @ barney.ife.no A: 15 Jan 93 / Ab: 15 Jan 93 / PP / 100 / NKR 800.-; incl. board, lodge; incl. proc.

14- 16 Crystal Growth: General Disc. Glasgow, UK Y.A. Fish, Royal Soc of Chem., Burlington House, London W1V OBN, UK $+44(71)$ $4378656 / 7341227$

16-23 Emittance Preservation in Linear Colliders: Int. Workshop Tsukuba, Japan K. Oide, Accel. Div., KEK, Nat. Lab. for High-Energy Physics, Oho, Tsukuba, Ibaraki 305, Japan +81 (298) $641171 / 64318$ urakawa @ jpnkekvm

18-24 Mathematical Foundations \& Numerical Methods in Transonic Fluids Oberwolfach, Germany Math. Forschungsinstifut, Albertstrasse 24 W-7800 Freiburg $\quad-/+49$ (761) 272698 
19-21 Power Sources: 18 th Int. Symp. Stratfordupon-Avon, UK POB 17, Leatherhead, Surrey KT22 9QB, UK A: 15 Mar $93 / \mathrm{PP} /<250$

19-22 The Institute of Physics Cong.: Highe Education Issues; Vacuum, Plasma \& Surface Technology; Spectroscopy Conf.; 20th. Ann. Plasma Physics Brighton, UK Meetings \& Cong. Office, Inst. of Physics, 47 Belgrave Sq., London SW1X $8 Q X$, UK $\quad+44(71) 2356111 / 2596002$

19-22 Integrated Optics: European Conf. (ECIO '93) Neuchâtel, Switzerland O. Parriaux, Swiss Centre for Electronics \& Microtechnology, CP 41 $\mathrm{CH}-2007$ Neuchâtel +41 (38) $205111 / 205580$ ICO, SPIE

19-22 Indium Phosphide \& Related Materials: 5th Int. Conf. (IPRM-5) Paris, France IEEE Inc. Conf. Cordination, 345 E 47 th St. New York, NY 10017, USA

19-23 Mathematical Methods \& Supercomputing in Nuclear Applicns.: Joint Int. Conf. Karlsruhe, Germany H. Küsters, KfK GmbH, Postfach 3640 W-7500 Karlsruhe 1

+49 (72) 47822481 47824874 PP: 1 Jan 93 ENS, ANS

19-23 Applicn. of Isotope Techniques in Studying Past \& Current Environmental Changes in the Hydrosphere \& Atmosphere: Int. Symp. Vienna, Austria IAEA, Conf. Service Sect., POB 100, A-1400 Vienna $\quad+43(1) 2360 / 234564$

19-30 Superstrings: Spring School \& Workshop Trieste, Italy ICTP, POB 586, I-34100 Trieste +39 (40) $22401 / 224163$

20 - 23 Dense Z-Pinches: Int. Conf. London, UK H. Coppins, Blackett Lab., Imperial College, Prince Consort Rd., London SW7 2BZ, UK

21- 23 Polymer Sci. \& Technology Conf. (to honour I.M. Ward on his 65 th Birthday) Leeds, UK R.A. Duckett, IRC in Polymer Sci. \& Tech., Leeds Univ., Leeds LS2 9JT, UK

22 Application of SQUID's \& Superconducting Devices: IoP Low Temp. Group Brighton, UK Cong. \& Meetings Office, Inst. of Physics, $47 \mathrm{Bel}$ grave Sq., London SW1X 8QX, UK

$2356111 / 2596002$ iop @ ulcc.ac.uk

22 What Can Computer Algebra Do for You?: IoP Computational Physics Group Brighton, UK Inst. of Physics, 47 Belgrave Sq., London SW1X $8 Q$ X, UK +44 (71) $2356111 / 2596002$ iop @ ulcc.ac.uk 25-May 1 Low-Dim. Dynamics Oberwolfach Germany Math. Forschungsinst., Albertstrasse 24 W-7800 Freiburg $\quad-/+49$ (761) 272698

26 - 30 Magnetism \& Magnetic Mater.: Int. Conf. (INTERMAG) Vienna, Austria Courtesy Assoc. Inc., 65515 th St., NW, Suite 300 , Washington, DC 20005, USA +1 (202) $6395088 / 3476109$

26-30 Physics \& Instrumentation at Future Linear Colliders: Int. Workshop Kona, HI, USA F. Harris, Dept. of Physics \& Astronomy, Univ. of Hawaii, 2505 Correa Rd., Honolulu, HI 96822, USA +1 (808) $9562940 / 9562930$ fah @ uhhepg 27-30 Materials Sci. \& the Computational Methods: Int. Meeting (IMMS-4) Oran, Algeria H. Aourag, 22, rue Hoche, Sidi-bel-Abbes 22000 , Algeria $+213(7) 245251 / 242706 \quad$ A: 1 Jun 92 / PP, French; incl. proc.

27-30 Small-Angle Scattering: 9th Int. Meeting Saclay, France J.P. Cotton, Lab. Léon Brillouin, CEN-Saclay, F-91191 Gif-sur-Yvette Cédex $\quad-/+33$ (1) 69088261

28 - 30 Recent Adv. in Active Control of Sound \& Vibration: 2nd Conf. Blacksburg, VA, USA D. Williams, Mech. Engng Dept., Virginia Polytechnic Inst. \& State Univ., 203 Randolph Hall, Blacksburg, VA 24061 , USA $-1+1$ (703) 2319100

\section{MAY}

May Temperature \& Thermal Measurement 5th Symp. (TEMPMEKO 93) Karlovy Vary, Hungary IMEKO, POB 457, H-1371 Budapest $5+36$ (1) 153 $1562 / 1561215$ IMEKO

May Research Reactor Centres - Future Prospects: Seminar Jakata, Indonesia IAEA, Conf. Service Sect., POB 100, A-1400 Vienna + 43 (1) $2360 / 234564$

May Pressure Metrology from $1 \mathrm{kPA}$ to $1 \mathrm{GPa}$ : 2nd Int. Sem. Paris, France G.F. Molinar. IMGC, Srade delle Cacce, 73, I-10135 Turin +39 (11) $39771 / 346761$
May Hot \& Dense Nuclear Matter: NATO ASI Bodrum, Turkey W. Greiner, Inst. f. Theo. Physik, J.W. Goethe Univ., Postfach 1119 32, W-6000 Frankfurt-am-Main 11

May/June Int. Optometric \& Optical League Ann. Conf. Italy C. Whelan, IOOL, 10 Knaresborough PI., London SW5 OTG, UK

2.7 Quantum Electronics \& Laser Sci. Conf./ Conf. on Lasers \& Laser Optics (CLEO/QELS '93) Baltimore, MD, USA OSA, Meetings Dept., 2010 Massachusetts Ave. NW, Washington, DC 20036 USA +1 (202) $2238130 / 2231096$ OSA, IEEE 2-7 Physical Signatures of Boundary Layer Processes: NATO ARW Lillehammer, Norway A. Egeland, Physics Dept., Univ. of Oslo, POB 1048 . Blindern

3-5 Beam Instrumentation \& Diagnostics for Particle Accelerators: 1st European Workshop Montreux, Switzerland Ch. Parthé, CERN-SL $\mathrm{CH}-1211$ Geneva $23 \quad-/+41$ (22) 7822850 edipac @ cernvm.cern.ch

3-7 European Geophysical Soc. 19th Gen Ass. Wiesbaden, Germany EGS Office, Postfach 49. W-3411 Katlenburg-Lindau +49 (5556) 1440 4709 A: 15 Dec 92/ Ab: 1 Jan 93/PP / $2000 /$ DM 310. - (360.- after 9 Apr 93)

3-7 Perspectives in Nuclear Physics at Intermediate Energies: 6th Workshop Trieste, Italy ICTP, POB 586, I-34100 Trieste +39 (40) 22401 224163

3-7 Future Prospects in High-Energy Physics: ICFA Seminar Hamburg, Germany DESY, Notkestr. 85, W-2000 Hamburg 52 PP / 125 / inv.

4-6 Optical Fibre Sensors: 9th Conf. (OFS-9) Florence, Italy V. Cammelli, IROE-CNR, via Panciatichi, 64, I-50127 Florence + 39 (55) 4378512 4379569 Ab: 30 Nov $92 /$ PP SPIE

5-7 Vortices \& Heat Transfer: EUROTHERM Sem. Bochum, Germany K. Cornwell, Eurotherm, Dept. of Mechanical Engineering, Heriot-Wat Univ., Edinburgh EH14 4AS, UK $4495111 / 4513129$

5.7 Individual Monitoring of Radiation: Int Conf. Villigen, Switzerland H.G. Menzel, DG XII-D-3, CEC, Rue de la Loi, 200, B-1049 Brussels +32 (2) $2359298 / 2366256 \quad$ CEC

6-8 Super Collider: 5th Annual Ind. Symp. (IISSC-5) San Francisco, CA, USA P.E. Patterson, POB 171551, Can Diego, CA 92197, USA $+1(619) 4900164 / 4900138$

E 6 - 10 Using Energy in an Intelligent Way Europhysics Study Conference Greifswald, Germany R. Dekeyser. Dept. Natuurkunde, Celestijnenlaan 200D, B-3001 Heverlee +32 (16) $201015 / 201368 \quad$ fgbda03 @ blekul1 50 / inv. / no reg. fee; 100.- per day for accom.

9-13 Modern Developments \& Applicns. in Microbeam Analysis: 3rd European Workshop (EMAS' 93 ) Rimini, Italy A. Armigliato, CNR Istituto Lamel, via del Castagnoli, 1, I-40126 Bologna 9.14 Fluid-Particle Interactions: 3rd Conference Davos, Switzerland P. Foscolo, Chem. Eng. Dept. L'Aquila Univ., I-67100 L'Aquila $\quad-I+39(862)$ 432604

10-13 European Res. Networking in a Globa Context: 4th Joint European Networking Conf (JENC4) Trondheim, Norway RARE Secretariat Singel 466-468, NL-1179 AW Amsterdam +31 (20) $6391131 / 6393289$ raresec @ rare.nl

10 - 14 Measurement of Force \& Mass: 13 th Conf Helsinki, Finland IMEKO, POB 457, H-1371 Budapest $5+36$ (1) $1531562 / 1561215$ Ab: 13 May 92 / PP: 10 May 93

10-14 Intercalation Compounds: 7th Int. Symp. (ISIC-7) Louvain-la-Neuve, Belgium J.-P. Issi Unité PCPM, Univ. of Louvain-la-Neuve, 1, pl. Croix du Sud, B-1348 Louvain-la-Neuve

10-14 Holography, Correlation Optics \& Recording Materials: Int. Conf. Chernovtsy, Ukraine O.V. Angelsky, Dept. of Correlation Optics, Chernovtsy Univ., 2 Kotsyubinsky St., 274012 Chernovtsy Ukraine +7 (03722) $44730 / 41314$ SPIE

10-21 Middle Atmosphere Interactions: Workshop Trieste, Italy ICTP, POB 586, 1-34100 Trieste +39(40) 22401/224163
11-15 Advances in Solar Physics: 7th European Meeting on Solar Physics Catania, Italy G. Belvedere, Oss. Astrofisico di Catania, viale A. Doria, 6, I-95125 Catania +39 (95) $330533 / 330592$ solar93 @ astrct.astro.it A: 31 Jan 93 / PP / $200 /$ SUS 70.-; 50.- IOM's; 10.- students

11-15 Heavy-Ion Physics: Int. School/Seminar Dubna, Russia Y.T. Oganessian, JINR, Lab. of Nuclear Reactions, Head Post Office, POB 79. 141980 Dubna, Moscow Region $\quad-/+7$ (095) 9752381 kalpak @ ljar10.jinr.dubna.su A: 15 Dec 92 / Ab: 15 Feb 93 / PP / inv. / \$US 200.-; incl. proc. 13-14 Microlens Arrays Teddington, UK European Optical Soc., BP 147, F-91043 Orsay Cédex IOP, IEEE

16-18 Dannish Phys. Soc. Spring Meeting Rødby, Denmark $\quad$ B. Andersen, Physics Lab., Univ. of Copenhagen, Universitetsparken 5, DK-2100 Copenhagen $\varnothing \quad+45$ () $35321818 / 35320460$ kuflba @ vm.uni-c.dk / 200

16-21 Computer Coupling of Phase Diagrams \& Thermochemistry: 22nd Ann. Int. Conf. (CALPHAD22) Barcelona, Spain N. Clavaguera, Fac de Fisica, Univ, de Barcelona, Avda Diagonale 647. E-08028 Barcelona

16-22 Mathematical Problems in Viscoelastic Flows Oberwolfach, Germany Math. Forschungsinst., Albertstr. 24, W-7800 Freiburg - - +49 (761) 272698

16-22 Small-Angle X-Ray Scattering: Int. Conf. Como, Italy Centro di Cultura Scientifica, "A. Volta", Villa Olmo, Via Cantoni, 1, 1-22100 Como +39 (31) $572213 / 573395$

17 - 21 Particle Accelerator Conf. Washington, DC, USA A. Quarrie, CEBAF, MS 12-A1, 12000 Jefferson Ave. Newport News, VA 23606, USA +1 (804) $2496377 / 2497398 \quad$ quarriea @ cebaf.gov Ab: 4 Dec 92/PP: 17 May 93 APS, IEEE 20-22 Deutsche-Bunsen Ges. f. Physikalische Chemie Hauptversammlung 1993 Leipzig, Germany DBG f. Physikalische Chemie, W-6000 Frankfurt-amMain $90+49(69) 7917201 / 7917322 \quad \mathrm{IUCr}$ 23-26 Toward Next-Generation Light Water Reactors: ENS Topical Meeting The Hague, The Netherlands ENS, POB 5032, $\mathrm{CH}-3001$ Bern +41 (31) $216111 / 229203$

23-27 Polarized Ion Sources \& Polarized Gas Targets: Int. Workshop Madison, WI, USA L.W. Anderson, Physics Dept., University of Wisconsin 1150 Univ. Ave., Madison, WI 53706, USA +1 (608) $2626555 / 2623598 \%$ madspin @ wiscnuc 24-27 Radioactive Nuclear Beams: 3rd Int. Conf. East Lancing. MI, USA D.J. Morrissey, Cyclotron Lab., Michigan State Univ., East Lansing, MI 48824, USA morrissey (@) msunscl

24-27 Measurement Assurance in Dosimetry: Int. Symp. Vienna, Austria IAEA, Conf. Service Sect., POB 100, A-1400 Vienna +43 (1) 2360 234564

24-28 Physical Concepts \& Materials for Novel Optoelectronic Device Applicns.: 2nd Int. Symp. Trieste, Italy Direct Communications $\mathrm{GmbH}$, Xantener Str. 20, W-1000 Berlin $15 \quad+49$ (30) 8815047 I $8822028 \quad$ Ab: 2 Nov 92 / P: 26 Apr 93 / $\approx \mathrm{DM} 650$. SPIE, EOS

24- 28 Surface Physics: 6th Symp. Prague, Cze cho-Slovakia J. Koukal, Inst. of Physics, Cukrovarnická 10, CS-162 00 Prague 6 3123184 IUPAP

24- 28 Heat \& Mass Transfer Under Plasma Conditions: ICHMT Int. Seminar Estoril, Portugal P. Fauchais, Fac. de Sciences, Univ. de Limoges, 123, ave. Albert-Thomas, F-87060 Limoges Cédex +33 () $55457435 / 55457211$

24-28 Crystallography of Biological Macromolecules: 3rd European Workshop Como, Italy Centro di Cultura Scientifica, "A. Volta", Villa Olmo Via Cantoni, 1, I-22100 Como +39 (31) 572213 / 573395

24-28 High Intensity Electron Sources: Workshop Legnaro, Italy M. Venco, Lab. Naz. di Legnaro, via Romea, 4, I-35020 Legnaro +39 (49) $8292328 / 641925$ venco @ legnaro.infn.it 24-28 Diffractometry: Int. Conf. Warsaw, Poland M. Pluta, Central Lab. of Optics, Ul. Kamionkowska 18, PL-03-805 Warsaw +48 (22) 184405 133265 SPIE 
24-28 New Physics at New Experiments: 16th Int. Warsaw Meeting on Elementary Particle Physics Kazimierz, Poland Z. Adjuk, Inst. of Theo. Physics, Warsaw Univ., ul. Hoza 69, PL-00-681 Warsaw +48 (2) $6283396 / 219475$ ajduk @ plearn A: 31 Mar $93 /$ Ab: 15 Apr 93/PP: 30 Sep $93 / 100 /$ \$US 200.-; incl. board, lodge

25-26 Rencontre Soc. Française de Thermiciens (SFT 93) Pau, France SFT, 3, rue Henri-Heine, F-75016 Paris +33 (1) $42245935 /$ 44304198

25-27 Artificial Neural Networks: 3rd. Int. Conf. Brighton, UK $\quad$ S. Griffiths, IEE Conf. Services, Savoy Place, London WC2R OBL, UK $\quad+44(71)$ $2401871 / 4973633 \quad$ IOP, IEE

25 - 29 High Power Particle Beams: 9th. Int. Conf. $\begin{array}{ll}\text { (Beams '93) Washington, DC, USA } & \text { D. Mosher, }\end{array}$ (Beams '93) Washington, DC, USA D. Mosher,
Naval Res. Lab., Univ. of Maryland, POB1077, Oxon Naval Res. Lab., Univ. of Maryland, POB1077, Oxon
Hill, MD 20750, USA +1 (202) $4048360 / 4048110$

26- June 2 Fundamental Aspects of Clusters: European Res. Conf. in Physics Elounda, Crete, Greece J. Hendekovic, ERC, ESF 1, quai Lezay Marnésia, F-67000 Strasbourg Cédex +33088767135/88366987

27 - 28 Belgian Phys. Soc. Gen. Scientific Assembly Leuven, Belgium J. Ingels, Belgian Inst. for Space Aeronomy, Ringlaan 3, B-1180 Brussels

29 - June 3 Nuclear Astrophysics: European
Res. Conf. in Physics Heraklion, Greece
J. Hendekovic, ERC, ESF, 1, quai Lezay Marné-
sia, F- 67000 Strasbourg Cédex $\quad+33$ ()
88767135 / $88366987 \quad$ A: 15 Feb 92 /
Ab: 15 Feb 92 / NP/ 100; incl. board, lodge CEC

31 - June 4 Physics Computing '93: 5th Joint EPS APS Int. Conf. on Physics Computing (PC'93) Albuquerque, NM, USA M. Scanlan, APS, $335 \mathrm{E}$ 45th St., New York, NY 10017, USA +1 (212) $6827341 / 6872532$ Ab: 5 Mar $93 \quad$ AIP

\section{JUNE}

June Geometric \& Topological Methods in Theo. Physics Lyon, France C. Roger, Lab. de Géomérie et Analyse, Univ. Claude Bernard, 43 , blvd du 11 novembre 1918, F-69622 Villeurbanne Cédex

1-5 Deutchen Ges. f. angewandte Optik 94 Tagung Wetzlar, Germany W. Vollrath, Leica Mikroskopie und System GmbH, Postfach 2040 , W-6330 Wetzlar $1+49$ (6441) $293399 /$ -

2. 4 Coupled Transport Processes \& Phase Transitions: Lars Onsager Symp. Trondheim, Norway Onsager Symp., NTH, N-7034 Trondheim - / +47 (7) 591676 onsager93 @ imf.unit.no

2-5 Insulating Films on Semiconductors: 8th Biennial Conf. (INFOS'93) Delft, The Netherlands C.H. Klein, DIMES, Delft Univ. of Tech., Postbus 5053, NL-2600 GB Delft + +31 (15) 783868 622163 chklein @ dimes.tudelft.nl Ab: 15 Jan 93 6-10 American Astro. Soc. 182th Meeting Berkeley, CA, USA S. Bowyer, Univ. of California, Berkeley, CA, USA

6-10 Thermal Energy: 1st Int. Cong. Marrakech, Tunisia A. Mir, Fac. of Science Semlalia Cadi Ayyad Univ., BP 515, Marrakech

6-10 Heavy Flavour Physics: 5 th Int. Symp. Montreal, Canada D.B. MacFarlane, Physics Bldg. McGill Univ., 3600 University St., Montreal, Quebec H3A 2TB, Canada +1 (514) $3986507 / 3983733$ hep @ physics.mcgill.ca

6.12 Materials Processing in High Gravity Potsdam, NY, USA L. Regel, Int. Center for Gravity Materials Sci. \& Applcns., Potsdam, NY 13699. 5700, USA

7-9 Plasma Science: 20th IEEE Int. Conf. (ICOPS-20) Vancouver, BC, Canada A. Ng Physics Dept, Univ, of British Columbia, Agricultural Rd, Vancouver, BC,V6T 2A6, Canada $2283191 / 2283853$

7.11 Radionuclide Metrology \& its Applcns Cont. \& Int. Symp. (ICRM'93) Teddington, UK P. Christmas, NPL, Teddington, Middlesex TW11 OLW, UK +44(81) $9436317 / 9432155$

7-13 Physics \& Chemistry of Fullerenes: NATO ARW Aghia Pelaghia, Crete, Greece K. Prassides, School of Chem. \& Molecular Sci. Sussex Univ. , Falmer, Brighton BN1 9QJ, UK
8-10 Norwegian Phys. Soc. Physics Meeting Oslo, Norway J.A. Holtet, Physics Dept., Univ, of Oslo, POB 1048, Blindern, N-0316 Oslo $3+47$ (2) 855669 /.

8-11 Real-Time Applicns. in Nuclear, Particle \& Plasma Physics: 6th IEEE Conf. Vancouver, BC Canada R. Risto, TRIUMF, 4004 Westbrook Mall Vancouver, BC, V6T 2A3, Canada $2221047 / 2228325$

8 - 12 Pattern Formation \& Lattice Gas Automata: NATO ARW Waterloo, Canada R.E. Kapral Chemistry Dept., Univ. of Toronto, Toronto, Ontario, M5S 1A1, Canada

8-12 Evolution of the Universe \& the Observational Quest: 37th Yamada Conf. Tokyo, Japan K. Sato, Sci. Fac., Tokyo Univ., Bunkyo-ku, Tokyo 113, Japan yamaconf\%tkyvax.hepnet@ lbl

9-10 Neutrino Astronomy: Disc. Meeting London, UK Royal Soc. of Chem., Burlington House, London W1V OBN, UK +44(71) $4378656 / 7341227$ 13- 18 Carbon: Biennial Conf. Buffalo, NY, USA D. Chung, Comp. Mater. Res. Lab., Furnas Hall State Univ, of NY, Buffalo, NY 14260, USA +1 (716) 6362520 /.

13-16 Canadian Assocn. of Physicists Cong Burnaby, Canada F.M. Brûlé, Suite 903 151 Slater St., Ottawa, Ontario K1P 5H3, Canada +1 (613) $2373392 / 2381677$

13-17 Vibration at Surfaces: 7th Int. Conf. Santa Margherita, Italy M. Rocca, Dip. di Fisica, via Dodecaneso, 33, l-16124 Genoa +39(10) $3536324 / 314218 \quad$ A: 1 Mar 92 / PP / LIT 280 000.-; 250 000.- IOM's; 130 000.- students 13-18 Laser Spectrosocpy: 11th Int. Conference Hot Springs, VA, USA T.F. Gallagher, Phys. Dept. University of Virginia, Charlottesville, VA 22901, USA IUPAP

14-16 Thermal Management in Electronic Systems: EUROTHERM Sem. Delft, The Netherlands C.J. Hoogendoorn, Applied Physics Dept., Delft Univ. of Tech., Lorentzweg 1, NL-2628 CJ Delft +31 (15) $784404 / 783251$

14- 17 Coherent States: Int. Symp. Oak Ridge TN, USA D.H. Feng, Drexel Univ. feng @ duvm 14-18 Perspectives in Nuclear Structure: Int Meeting Copenhagen, Denmark U. Hølm, Niels Bohr Inst., Blegdamsvej 21, DK-2100 Copenhagen +45 (42) $373516 / 42373516$ bh @ nbital.nbi.dk 14- 18 Formation of Semiconductor Interfaces 4th Int. Conf. (ICFSI-4) Jülich, Germany B. Lengeler, Forschungszentrum Jülich, Postfach 1913 W-5170 Jülich +49(2461) $613008 / 614666$ A: 30 Apr 93 / Ab: 15 Jan 93 / PP: 14 Jun $93 / 250$ DM 500.-; 200.- students; incl. proc. IUPAP

14- 18 Open Plasma Confinement Systems for Fusion: Int. Conf. Novosibirsk, Russia A.N. Strinsky, Budker Inst. for Nuclear Physics, CIS-630090 Novosibirsk

14-18 Safe Management \& Disposal of Nuclear Waste: Int. Conf. (Safewaste '93) Avignon, France SFEN, 48, rue de la Procession, F-75724 Paris Cédex +33(1) $44496000 / 44496011$

14-18 Dynamical Aspects of Nuclear Fission: Int. Symp. Smolenice, Czecho-Slovakia J. Kristiak, Inst. of Physics, Slovak Acad. of Sci., Dubravska cesta 9, CS-842 28 Bratislava +42 (7) 3782135 376085 fyzikris@ savba.cs.eunet IUPAP 14-19 Molecular Spectroscopy: 48th Symposium Columbus, $\mathrm{OH}, \mathrm{USA} \quad$ Ohio State Univ., Physics Dept, $174 \mathrm{~W}$ 18th Ave, Columbus, $\mathrm{OH} 43210$, USA 14-19 Groups of Lie Type \& Their Geometries Int. Conf. Como, Italy Centro di Cultura Scientifica, "A. Volta", Villa Olmo, Via Cantoni, 1, I-22100 Como +39 (31) $572213 / 573395$

14- 25 Nanophase Materials - Synthesis, Properties, Applens.: NATO ASI Marathon, Greece G.C. Hadjipanayis, Physics \& Astronomy Dept., Univ. of Delaware, Newark, DE 19716, USA

15 - 20 Fund, Aspects of Surface Sci. European Res. Conf. in Physics Davos, Switzerland J. Hendekovic, ERC, ESF, 1, quai Lezay Marnésia, F-67000 Srasbourg Cédex +33( $88767135 / 88366987 \quad$ A: 15 Mar 93 Ab: 15 Mar 93 / NP / 100; incl. board, lodge ESF, CEC
20-24 American Nuclear Society Ann. Meeting Las Vegas, AZ, USA ANS, 555 N. Kensington Ave. La Grange Park, IL 60525, USA +1 (708) 3526611 I 3526464

20-24 Ultra-Relativistic Nucleus-Nucleus Collisions: 10th Int. Conf. (Quark Matter '93) Bor Laenge, Sweden B. Bring, Dept. of Cosmic \& Subatomic Physics, Univ. of Lund, Solvegatan 14, S-223 62 Lund $-1+46$ (46) 104709 kosu_hansak @ lucas.lu.se 20-24 Positron \& Positronium Chemistry: 4th Int. Workshop (PPC-4) Obernai, France J.-C. Abbe CNR, Lab. de Chimie Nucléaire, BP 20, F-67037 Strasbourg Cédex +33088286405/88280990 A: 31 Mar 93/ Ab: 31 Jan 93 / PP: 20 Jun 93

20-25 The Sun as a Variable Star-Solar \& Stellar Irradiance Variations: IAU Coll. 143 Boulder CO, USA J. Pap, JPL, Caltech, 4800 Oak Grove Drive, Pasadena, CA 91109-8099, USA

20-26 Chaos \& Complexity: V Rencontres de Blois Château de Blois, France J. Trân Thanh Vân, LPTHE, Bât. 211, Univ. de Paris-Sud, F-91405 Orsay Cédex +33 (1) $69285135 / 69288659 \quad \mathrm{~A}: 1 \mathrm{Ma}$ 92 / Ab: 1 Mar 92 / PP / 180 / inv. / =FF 4500.-; incl. board, lodge; incl. proc. CEC, CNRS, CEA, NSF 21-25 Math/Chem/Comp'93: 8th Course \& Conf Dubrovnik, Croatia A. Graovac, Ruder Boškovic Inst., POB 1016, 41001 Zagreb, Croatia +38 (41) $4525111 / 425497 \quad$ ante.graovac @ olimp.irb.ac.mail.yu A: 15 Apr 93 / Ab: 15 May 93 PP: 15 Sep 93 / \$US 150.-; 15.- students

21-26 Infrared Astrophysics: Topical Conference (CIRP-5: 5th Int. Conf. on Infrared Physics) Ascona Switzerland F.K. Kneubühl, Infrared Physics Lab. HPF, ETH, Hönggerberg, $\mathrm{CH}-8093$ Zurich +41 (1) $3772340 / 3715989 \quad$ Ab: 1 Feb $93 /$ PP: 1 May 93 / SFR 330 . SGAA, SSP

21.9 July Strongly Correlated Electron Systems: Miniworkshop Trieste, Italy ICTP, POB 586 1-34100 Trieste +39 (40) $22401 / 224163$

21 - Sept. 3 Cond. Matter, Atomic \& Molecular Physics: Res. Workshop Trieste, Italy ICTP POB 586, I-34100 Trieste +39 (40) 22401 $224163 \quad$ A: 28 Feb 93

22 - 25 Fermi Liquids vs Non-Fermi Liquids: Adriatico Res. Conf. Trieste, Italy ICTP, POB 586 I-34100 Trieste + $39(40) 22401 / 224163$

22-27 Physics in the Ukraine: Conf. Kiev, Ukraine A.G. Sitenko, Inst. for Theo. Physics, 252143 Kiev, Ukraine nmakovsky @ glas.apc.org 24-30 Multiphoton Processes: 6 th Int. Conference (ICOMP VI) St. Foy, Canada S.L. Chin, Physics Dept., Laval Univ., Pavillon Vachon, Quebec, G1K 7P4, Canada $\quad-/+1(418) 6563738$ IUPAP

$26-30$ 15th ICA Cong. Trondheim, Norway

26-July 2 Experimental Gravitation: Int. Symp. Nathiagali, Pakistan M. Karim, Physics Dept. St. John Fisher Coll., 3690 E. Ave., Rochester, NY 14618, USA +1 (716) $3858365 / 3858129$ krim@uordbv IUPAP, UNESCO

27 - 30 Sci. \& Technology of Fullerenes: 1st Int. Interdisciplinary Conf. Santa Barbara, CA, USA K. Cavellero, Pergamon Seminars, 660 White Plains Rd., Tarrytown, NY 10591-5153, USA +1 (914) $3332550 / 3332468$

27-30 Prog. in Electromagnetics Res.: Symp. Pasadena, CA, USA J.J. van Zyl, MS180-404, JPL, 4800 Oak Grove Drive, Pasadena, CA 91109 , USA +1 (818) $3549311 / 3934468$

27 - July 1 Mag. Resonance in Bio. Systems 16 th Int. Conf Veldhoven, The Netherlands A. Manders, Postbus 140, NL-5500 AC Veldhoven

27- July 2 Indoor Radon Remedial Actions: 1st Int. Workshop Rimini, Italy M. Olast, DG XII-D-3, CEC, Rue de la Loi, 200, B-1049 Brussels +32 (2) $2350723 / 2366256$

28-29 Plastic Optical Fibres \& Applicns.: 2nd Int. Conf. (POF '93) The Hague, The Netherlands c/o AKM, POB 6, CH-4005 Basel

28 - July 1 Laserdiagnostik für industrielle Prozesse: Bunsen-Diskussionstagung Heidleberg, Germany Deutschen Bunsen-Ges. f. Physikalische Chemie, W-6000 Frankfurt-am-Main 90 +49 (69) $7917201 / 7917322$

28- July 2 High Pressure \& Technology: Joint APS/AIRPAT Conf. Colorado Springs, CO, USA A. Roach, LANL, MS P915, Los Alamos, NM 87545 USA +1(505) $6656277 / 6653407$ Ab: 15 Feb 93 / PP / 600 / \$US 300.-; incl. proc. 
28 - July 2 Particles \& Nuclei: 13th Int. Conf. (PANIC 93) Perugia, Italy C. Schaerf, Physics Dept, II Univ. of Roma, via E. Carnevale, I-00173 Rome+ +39 (6) $79794560 / 2040309$ schaerf (1) roma2.infn.it IUPAP

28- July 2 Shock Compression of Cond. Matter Physics (jointly with AIRPAT) Colorado Springs, CO, USA APS, 335 E. 45th St., New York, NY 10017, USA $\quad+1$ (212) $6827341 / 6872532$ 28 - July 2 Atomic \& Nuclear Clusters: 20th Int. Meeting Santorini, Greece G.S. Anagnostatos, Inst. of Nuclear Physics, POB 60 228, GR-15310 Aghia Paraskevi +30 (1) $5518770 / 6511215$ geras@ grathdem A: 15 Feb 93 / Ab: 1 Apr 93 / PP / 150 / SUS 400.-; 350.- students; incl. proc.

28 - July 2 High Latitude Optics: Int. Symposium Tromsø, Norway Direct Communications $\mathrm{GmbH}$, Xantener Str. 22, W-1000 Berlin $15 \quad+49$ (30) $8815047 / 8822028$ Ab: 9 Nov 92 / PP: 31 May 93/ DM 500.- EOS, SPIE

28 - July 2 Hamiltonian Mechanics - Integrability \& Chaotic Behaviour: NATO ARW Torum, Poland J. Siemens, Maths Dept., Univ. of the Aegean, GR-83200 Samos

28 - July 2 Nonlinear Acoustics: 13th Int. Symp. (ISNA-13) Bergen, Norway H. Hobæek, Physics Dept., Univ. of Bergen, Allégaten 55, N-5007 Bergen $-1+47$ (5) 318334 IUPAP 28 - July 3 Int. Soc. for Theo. Chem. Physics: 1st Cong. Girona, Spain R. Carbó, Inst. de Quimica Computacional, Fac. de Ciencias, Univ., Placa del Hospital, 6 +34 (72) $226366 / 216406$ quantum @ ccegg.uab.es A: 31 Apr 93 Ab: 31 May 92 / PP: 31 Jul $93 / 250$ / SUS 300.-; 200.- students

28 - July 3 Future of Nuclear Spectroscopy: Int Conf. Aghia Pelaghia, Crete, Greece C.A. Kalfas, Inst. of Nuclear Physics, РОВ 60 228, GR-15310 Aghia Paraskevi +30 (1) $5518770 / 6511215$ A: 18 Feb 93 / Ab: 1 Apr 93 / PP / 150 / ECU 240.-incl. proc.

29- July 9 Adaptive Optics for Astronomy: NATO ASI Cargese, France D.M. Alloin, URA 173 du CNRS, Obs, de Paris, F-92195 Meudon

30 - July 2 European Fibre Optic Communications \& Networks: 11 th Ann. Conf. (EFOC\&N '93) The Hague, The Netherlands C/O AKM, POB 6 $\mathrm{CH}-4005$ Basel +41 (61) $6918888 / 6918189$

\section{JULY}

July 3-16 Chaos - Towards the Next Century: NATO Adv. Res. Workshop Como, Italy Centro di Cultura Scientifica, "A. Volta", Villa Olmo, via Cantoni, 1, 1-22100 Como +39 (31) 5722 13/573395 5.9 Liquid-Particle Interactions in Suspension Flow Grenoble, France G. Cognet, ENSGIINPG, 46, ave. Félix-Viallet, F-38031 Grenoble Cédex $-1+33$ () 76574793 IUTAM

5 - 9 Ion Beam Analysis: 11 th Int. Conf. (IBA-11) Balatonfüred, Hungary K. Lang, Lorand Eötvös Phys. Soc., POB 433, H-1371 Budapest +36 (1) $2018682 / 2019760$ PP KFKI, IUPAP 5-9 Energy Systems \& Ecology: Int. Conf. (ENSEC '93) Cracow, Poland Inst. of Thermal Technology, University of Silesia, Konarskiego 22, PL-44-101 Gliwice

5-9 Optical Memory \& Optical Data Storage: IEEE/OSA Joint Int. Symp. Maui, HI, USA OSA, Meetings Dept., 2010 Massachusetts Avenue, NW, Washington, DC 20036, USA

5-9 French Phys. Soc. General Cong. (SFP93) Toulouse, France A. Rocher, CEMES/LOE du CNRS, 29, rue de Marvig, F-31055 Toulouse +33() $62257800 / 62657899$

cemes.fr A: 15 Mar 93 / PP / 1000, French only / FF 1250.-: 200.- students

$6-8$ Ultrasonics Int. '93 Vienna, Austria J. Herriot, Meetings Mgt, Straight Mile House, Tilford Rd. Rushmoor +44 (25125) 54 14/121 01

6-9 European Optical Soc. Ann. Meeting Saragossa, Spain European Optical Soc., BP 147, F-91043 Orsay Cédex

6-9 Neutral Currents: 20 Years Later Paris, France U. Nguyen-Khac, Lab. PNHE, Ecole Polytechnique, F-91128 Palaiseau Cédex +33 (1) $69334136 / 69333002$ nc93@ frcpn11

$6-9$ Scattering from Surfaces: Adriatico Res. Conf. Trieste, Italy ICTP, POB 586, I-34100 Trieste $\quad+39(40) 22401 / 224163$
6-9 23rd AIAA Fluid Dynamics, Plasmadynamics \& Laser Conf. + 11 th AIAA Computational Fluid Dynamics Conf. + 28th AIAA Thermophysics Conf. Orlando, FL, USA AIAA, Meetings Dept., 370 L'Enfant Promenade SW, Washington, DC 20024, USA

11-15 Health Physics Soc. Ann. Meeting Atlanta, GA, USA Health Physics Soc., 8000 Westpark Drive, Suite 400, McLean, VA 22102, USA

11-16 Optical \& Optoelectronic Applied. Sci. \& Engng.: 38 th Int. Symp. San Diego, CA, USA SPIE, POB 10, Bellingham, WA 98227-0010, USA +1 (206) $6763290 / 6761445$

11-23 Int. Assocn. of Meteorology \& Atmospheric Physics: 6th Sci. Assembly + Int. Assocn. of Hydrological Sciences: 4th Science Assembly Yokohama, Japan c/o Sankei Conv., Sankei Bldg 10F, 1-7-2 Otemachi, Chiyoda-ku, Tokyo 100

12-15 Optics \& Dynamics of Polymers: 33rd Microsymp. Prague, Czecho-Slovakia P. Cefelin, Inst. of Macromolecular Chem., Heyrovskeho namesti 2, CS-162 06 Prague 6

SFR 250.-

12-16 X-Ray \& Inter-Shell Processes: 16 th Int. Conf. (X'93) Debrecen, Hungary D. Berényi, Inst. of Nuclear Research, POB 51, H-4001 Debrecen +36 (52) $17266 / 13945 \quad 1775 \mathrm{kad}$ @ ella.uucp A: 4 May 93 / PP / 450 / \$US 280.-; 265.- IOM's; incl. proc. IUPAP

13 - 16 European Group for Atomic SpectroD. Leclèr, Lab. de Spectroscopie Atomique, ISMRa, Blvd. Maréchal Juin, F-14050 Caen Cédex PP / 350

14 - 16 The Bubble Chamber \& Its Rôle in Particle Physics Geneva, Switzerland S.M. Tracy, CERN-DG, CH-1211 Geneva 23 +41 (22) $7823011 / 7672724$ PP/ $=$ SFR 300 .; incl. proc. 14-17 Positron Interactions with Atoms, Molecules \& Clusters: Int. Workshop Bielefeld, Germany W. Raith, Fak. f. Physik, Bielefeld Univ. Postfach 100131 , W-4800 Bielefeld 1

DM 150 .-; incl. proc.

$15-17$ (e, 2e) Collisions, Double Photoionization \& Related Processes: Int, Symp. (XVIIII ICPEAC Sat. Meeting) Paris, France A. Lahmam-Bennani, Lab. de Collisions Atomiques et Moléculaires, Univ. de Paris-Sud, Bâtiment 351, F-91405 Orsay Cédex I +33 (1) 69417671 $=\mathrm{FF} 800$.

16-21 Metallic Alloys - Experimental \& Theo. Perspectives: NATO ARW Boca Raton, FL, USA J.S. Faulkner, Physics Dept., Florida Atlantic Univ. Boca Raton, FL 33431 , USA

16- 21 Light \& Information: Int. Conf. on Physics Education Braga, Portugal L Chainho Pereira, Univ. do Minho, Largo do Paco, P-4719 Braga Codex +351 (53) $61234 / 616936$ IUPAP, GIREP

17-19 Fast Ion-Atom Collision Processes: 5th Workshop Debrecen, Hungary $\quad$ D. Berenyi, Inst. of Nuclear Res., POB 51, H-4001 Debrecen +36 (52) $17266 / 13945$ h1775kad @ ella.uucp A: 30 Apr 92 18 - 23 Defects in Semiconductors: 17 th Int. Conf. Gmunden, Austria W. Jantsch, Inst. für Experimentalphysik, Johannes-Kepler-Univ., A-4040 Linz +43 (732) $24689641 / 24689666 \quad k 340830$ (a) aliiku11 A: 15 Jun 93 / Ab: 15 Feb 93 / PP: 18 Jul 93/450/AS 3000.-; 2000.- students; incl. proc.

18-24 Dynamic Systems: Tagung Oberwolfach, Germany Math. Forschungsinst., Albertstrasse 24. W-7800 Freiburg $\quad-1+49$ (761) 272698

19-23 Nuclear \& Space Radiation Effects: 30th Ann. Int. Conf. (NSREC-30) Snowbird, UT, USA P. Dressendorfer, Sandia Nat. Lab., POB 5800, Albuquerque, NM 87185, USA +1 (505) 8445373

$19-23$ Nonlinearity \& Chaos in Engineering Dynamics London, UK J.M.T. Thompson, Dept. of Civil Engineering, London Univ., Gower St., London WC1E 6BT, UK $\quad-/+44$ (71) 3835519 IUTAM 19-23 Nuclear Quadrupole Interaction: 12th Int. Symp. Zurich, Switzerland D. Brinkmann, Physik-Inst., Zurich Universităt, Schönberggasse 9 , $\mathrm{CH}-8001$ Zurich brinkman @ physik.unizh.ch

19-23 Solar Surface Magnetism: NATO ARW Soesterberg. The Netherlands R.J. Rutten, Sterrekundig Inst., Utrecht Univ., Postbus 80.000 , NI-3508 TA Utrecht
19-30 Cosmic Rays: 23rd Int. Conf. (ICR-23) Calgary, Canada D. Venkatesan, Physics Dept. Univ. of Calgary, 2500 Univ. Drive, NW, Calgary T2N $1 \mathrm{~N} 4$, Canada $\quad-/+1(403) 2893331 \quad$ IUPAP 19-30 Mesoscopic Systems \& Chaos - A Novel Approach: Miniworkshop on Non-Linearity Trieste, Italy ICTP, POB 586, I-34100 Trieste +39 (40) $22401 / 224163 \quad$ / limited

19-30 Opportunities from New Radiation Sources: Miniworkshop on the Liquid State of Matter Trieste, Italy ICTP, POB 586, I-34100 Trieste +39 (40) $22401 / 224163$

$19-31$ Vortices in Superfluids: NATO AS

Cargese, France N. Bontemps, Lab. de Physique de la Matière Condensée, ENS, 24, rue Lhomond, F-75231 Paris Cédex 05

20-22 The Polymer Conf.

Cambridge, UK J. Herriot, Meetings Managt., Straight Mile House, Tilford Rd., Rushmoor $\quad+44$ (25125) 5414/121 01 21-27 Physics of Electronic \& Atomic Collisions: 18th Int. Conf. (XVIII ICPEAC) Aarhus, Denmark H. Knudsen, Inst. of Physics \& Astron. Aarhus Univ., DK-8000 Aarhus C +45 () 86128899 86120740 icpeac @ dfi.aau.dk Ab: 15 Feb 93/ PP / $200 /$ DKR 2000.-; 800.- students IUPAP

22 - 28 High-Energy Physics: Int. Europhysics Conf. Marseilles, France CNRS Luminy, Case 907, F-13288 Marseilles Cédex $9+33$ () $91269570 / 91414523$ eps @ marvax.in2p3.fr Ab: 1 May 93/PP: 1 Oct $93 /$ 900 / inv. / FF 1550.-; 1350.- IOM's \& students; incl. proc.

25-30 Shock Waves: 19th Int. Symp. (ISSW-19) Marseilles, France R. Brun, Case 321, Centre St. Jérôme, Université de Provence, F-13397 Marseilles Cédex $\quad-1+33091288322$

26 - 30 Controlled Fusion \& Plasma Physics: 20th European Conf. Lisbon, Portugal M.E. Masno, Centro de Fusão Nuclear, Inst. Sup. Técnicio, P-1096 Lisbon Codex +351 (1) $8473421 / 8499242$ PP: 10 May 93

26 - 30 Atomic Collisions in Solids: 15 th Int. Conf (ICACS-15) London, Canada W.N. Lennard, Physics Dept., Univ. of Western Ontario, London, Ontario N6A 3K7, Canada +1 (519) 6613283 6612033 wlennard (a) uwo.ca IUPAP 27 - 30 Mesoscopic Systems \& Chaos: Adriatico Res. Conf. Trieste, Italy ICTP, POB 586, 1-34100 Trieste $+39(40) 22401 / 224163$

28 - 31 Teachers in Maths, Physics \& Chemistry 15th Nordic Conf. Aarhus, Denmark Convention Bureau, Radhuset, DK- 8000 Aarhus

29-30 Ion-Atom Collisions: 13th Int. Seminar (ISIAC-13) Stockholm, Sweden R. Schuch, Manne Siegbahn Inst., Frescativaegen 24, S-104 05 Stockholm

$29-30$ Electron Collisions with Molecules, Clusters \& Surfaces: Int. Symp. London, UK L. Morgan, Computer Centre, London Univ., Egham Hill, Surrey TW20 OEX, UK A: 1 Dec $92 / P P$ / $<10 /=£ 50$.

29- 31 Polarization \& Correlation In Electronic \& Atomic Collisions: 7 th Int. Symp. (XVIII ICPEAC Sat. Meeting) Bielefeld, Germany R. Hippler. Fak. f. Physik, Bielefeld Univ., Postfach 100131 W-4800 Bielefeld $\quad-/+49$ (521) $1065244 \quad$ PP / DM 150.-: incl. proc.

31-Aug. 4 Ion Sources: 5th Int. Conf. (ICIS 93) $\begin{array}{ll}>\text { Beijing, PRC } & \text { Z. Weijiang, Inst. of Heavy }\end{array}$ Ion Physics, Beijing Univ., Beijing 100871, PRC

\section{AUGUST}

Aug. Dynamical Processes in Excited States of Solids: 9th Int. Conf. Boston, MA, USA R. Silbey, Chemistry Dept., MIT, 77 Mass Avenue, Cambridge, MA 02139, USA

Aug./ Sept. Phys. Sect., Union of Czecho-Slovak Mathematicians \& Physicists Ann. Meeting

Zilina, Czecho-Slovakia Inst. of Physics, CzechoSlovak Acad. of Sci., Na Slovance 2, CS-180 40 Prague $8 \quad+42$ (2) $8152910 / 8584569$

1-15 Perspectives in the Structure of Hadronic Systems Dronten, The Netherlands M. Oskam. NIKHEF-K, Postbus 41882, NL-1009 DB Amsterdam +31 (20) $5922163 / 5922165$ marike @ paramount.nikhefk.nikhef.nl A: $30 \mathrm{Apr} 93 / \mathrm{PP}$ / 80 / HFL 950.-; incl. board, lodge; incl. proc. 
1-6 Int. Union of Pure \& Applied Biophysics: 11th Triennial Cong. Budapest, Hungary J. Tigyi, Inst. of Biohysics, Medical Univ., H-7643 Pecs

1-6 Dynamics \& Reactive Systems: 14 th Int. Colloquium Coimbra, Portugal J. Campos, Centro de Thermodinamica, Universidad of Coimbra, P-3000 Coimbra

\section{2-5 Active \& Adaptive Optics: ICO-16 Sat.} Conf. Munich, Germany F. Merkle, ESO, KarlSchwarzschild-Str. 2, W-8046 Garching bei München +49 (89) $320060 / 3202362$ ESO, SPIE

2-6 Field Emission: 40th Int. Symp. Nagoya, Japan E.F. Okuyama, Applied Physics Lab. Nagoya Inst. of Tech., Gokiso-cho, Showa-ku

2-14 Turbulence, Weak \& Strong: NATO ASI Cargese, France P. Taebbeling, Lab. de Physique Statique, ENS, 24, rue Lhomond, F-75231 Paris Cédex 05

4-11 Low-Temperature Physics: Conf. (LT-20) Eugene, OR, USA R.J. Donnelly, Physics Dept, University of Oregon, Eugene, OR 97403-1274, USA +1 (503) $3464226 / 3464708$ IUPAP, APS 6- 19 Stochastic Analysis \& Applens. in PhysFunchal, Madeira, Portugal L. Streit, Univ. da Madeira, Ed. do Colegio Praca do Municipio, P-9000 Funchal

7-12 American Assocn. of Physics Teachers Summer Meeting Boise, ID, USA AAPT, 5112 Berwyn Rd., College Park, MD 20740, USA $-/+1$ (301) 3451857

8-12 American Assocn. of Physicists in Medicine: 35 th Ann. Meeting Washington, DC, USA AAPM, 335 E 45th St., New York, NY 10007, USA +1 (212) $6619404 /$.

8-13 Ferroelectricity: 8th Int. Meeting (IMF-8) Gaithersburg, MD, USA K. Kilmer, NIST, Admin. Bldg., Room A903, Gaithersburg, MA 20899, USA +1 (301) $9752858 / 9482067 \quad$ Ab: 15 Jan 93 / PP: 8 Aug 93 IUPAP

8-14 Applicns. of the Mossbauer Effect: Int. Conf. (ICAME '93) Vancouver, Canada J.G. Stevens, Univ. of North Carolina, Ashville, NC 28804, USA $\quad-/+1(704) 2516002 \quad$ A: 1 Jun 93 / Ab: 1 Mar 93/PP: 1 Jul 93 / \$US 325.- IUPAP 9-13. Int. Comm. for Optics 16 th Cong. - Optics as a Key to High Technology (ICO-16) Budapest. Hungary I. Bába, OPAKFI, Fö u. 68, H-1072 Budapest +36 (1) $2018964 / 2020452$ Ab: 1 Dec 92 / PP: 31 Mar 92 / SUS 200.- (240.- after 1 Jul 93); incl. proc.

SPIE, OSA, EOS, IUPAP

9-13 Scanning Tunneling Microscopy: Int. Conf. (STM '93) Beijing, PRC C. Bai, Chem. Inst., Chinese Acad. of Sciences, Beijing 100080, PRC +86 (1) $2568158 / 2569564$

9-13 Luminescence \& Optical Spectroscopy of Cond. Matter: 9th Int. Conf. (ICL'93) Storrs, CT, USA D.S. Hamilton, Physics Dept., Univ. of Connecticut, 2125 Hillside Road, Storrs, CT 06269-3046, USA $-1+1$ (203) 4863346 OSA, IEEE, IUPAP

10-13 Vortex Fluctuations in High- $\mathrm{T}_{c}$ Superconductors: Adriatico Res. Conf. Trieste, Italy ICTP, POB 586, I-34100 Trieste +39 (40) 224 01/22 4163 10-15 Lepton \& Photon Interactions: 16 th Int. Conf. Ithaca, NY, USA R. Galik, R. Newman Lab. of Nuclear Studies, Cornell Univ., Ithaca, NY 148538001, USA $\quad-1+1(607) 2544552$

11- 13 Photorefractive Materials, Effects \& Devices: Topical Meeting (PRM'93) Kiev, Ukraine OSA, Meetings Dept., 2010 Massachusetts Ave. NW, Washington, DC 20036, USA +1 (202) 2238130 \% 2231096 / Ab: 15 Mar 93

11. 14 Coherent Interactions in Semiconductors: NATO ARW Cambridge, UK R. Phillips, Cavendish Lab., Cambridge Univ., Madingley Rd., Cambridge $\mathrm{CB} 3 \mathrm{OHE}$, UK

15-21 Biomagnetism: 9th Int. Conference (ICB-9) Vienna, Austria L. Deecke, Allgemeines Krankenhaus der Stadt Wien, Währinger Gürtel 18-20, A-1090 Vienna $\quad-/+43$ (1) 404003141

16-19 Education \& Training in Optics: ICO-16 Sat. Conf. Pécs, Hungary Z. Füzessy, Physics Dept., Tech. Univ., H-1521 Hungary $\quad-/+36$ (1) 1812365 ICO, SPIE

16- 19 Structure of Surfaces: 4 th Int. Conference (ICSOS-4) Shangai, PRC S.Y. Tong, Physics Dept., Wisconsin Univ., Milwaukee, WI 53201, USA $-/+1(414) 2295589 \quad$ IUPAP
16 - 20 Noise in Physical Systems \& 1/f Fluctuations: 12th Int. Conf. (ICNF'93) St. Louis, MI, USA P.H. Handel, Physics Dept., Univ. of Missouri, St. Louis, MO 63121, USA +1(314) 5535021 / 5536152 c4621@ slvaxa.umsl.edu

16-20 Applicns. of Non-Linear Optics: 2nd Int. School \& Topical Meeting Prague, Czecho-Slovakia M. Kalal, Dept. of Physical Electronics, Fac. of Nucl. Sciences \& Phys. Engng., Czech TU, V. Holesovickach $2+42(2) 847786 / 847354 \quad$ tjejl @ cspuni12 A: 31 Jul $93 / \mathrm{PP} / 100 /=$ \$US $690 .-$; incl. board, lodge; incl. proc.

16-22 Hydrogen Bond Networks: NATO ARW Cargese, France M.C. Bellissent-Funel, CENSaclay, Lab. Léon Brillouin, F-91191 Gif-sur-Yvette Cédex

$17-19$ Neutron Scattering: 16 th IUCr Cong. Sat. $\begin{array}{lll}\text { Meeting Beidaihe, PRC } & \text { C. Ye, China Inst. of }\end{array}$ Atomic Energy, POB 275-30, Beijing 102413, PRC $-1+86$ (1) 9357008

21-29 Int. Union of Crystallography 16th Gen. Assembly \& Int. Cong. of Crystallography Beijing. PRC UCr, 5 Abbey Sq., Chester CH1 2HU, UK +44 (244) $4345431 / 344843$

22 - 29 History of Science: 19 th Int. Cong. Zaragoza, Spain Fac. de Ciencias, Univ, de Zaragoza, Ciudad Univ., E-50009 Zaragoza

23- 26 Synchrotron Radiation Instrumentation: Conf. Gaithersburg, MD, USA C. Clark, NIST, Physics Bldg. A253, Gaithersburg, MD 20899, USA 23-27 Free Electron Lasers: Int. Conf. (FEL'93) The Hague, The Netherlands M.J. van der Wiel, FOM Inst. for Plasma Physics, Postbus 1207, NL3430 BE Nieuwegein +31 (3402) 312 24/312 04 Ab: 1 May $93 /$ PP: 27 Aug $93 / 250 /=$ DFL 700.incl. proc. FOM

23-27 Int. Solar Energy Soc. World Solar Cong. Budapest, Hungary ISES, c/o Malev, POB 122, $\mathrm{H}-1367$ Budapest

23-27 Laser Anemometry - Advances \& Applicns: 5 th Int Conf. Veldhoven, The Netherlands L.A. Conf. 1993, Dr. Ter Braaklaan 1, NL-4002 WN Tiel +31 (3440) $15763 / 24103$

23- 28 European Magnetic Materials \& Applicns. Conf. (EMMA 93) Kosice, Czecho-Slovakia P. Sovák, Dept. of Exptl. Physics, Fac. of Sciences, Nám. Febr. vit'azstva 9, CS-04154 Kosice +42(95) $21128 / 22124$

23 - Sept. 3 Mechanical Props.: Working Party Trieste, Italy ICTP, POB 586, 1-34100 Trieste +39 (40) $22401 / 224163$

24-28 Few-Body Problems in Physics: 14th European Conf. Amsterdam, The Netherlands B.L.G. Bakker, Fac. of Physics \& Astrophysics, Free Univ., NL-1009 AJ Amsterdam blgbkkr@ nat.vu.nl

25 - Sept. 2 Int. Union of Radio Science (URSI) 24th Gen. Ass. Kyoto, Japan I. Heleu, URSI, c/o Univ. of Gent/LEA, Sint-Pietersnieuwstraat 41 , B-9000 Ghent+32 (91) $643320 / 643593$ heleu @ lea.rug.ac.be

28-Sept. 1 Silicon Molecular Beam Epitaxy: 5th Int. Symp. Makuhari, Japan Y. Shirak, Res. Center for Adv. Sci. \& Tech., Tokyo Univ., 4-6-1 Komba, Tokyo 153, Japan

29 - Sept. 1 Solid State Devices \& Materials: Int. Conf. (SSDM) Chiba, Japan Japan Soc. of App. Physics, Bus. Center for Academic Societies, Crocevia Bldg 2F, 3-23-1 Hongo

29-Sept. 2 Quantum Electronics: 11th Nat. Conf. Belfast, N. Ireland S. Swain, Dept. of Applied Maths \& Theo. Physics, Queen's Univ., Belfast, BT7 $1 \mathrm{NN}$. UK IOP

29 - Sept. 3 Reactor Dosimetry: 8th ASTM-EURATOM Symp. Vail, CO, USA D. Vehar, Div. 6452 , Sandia Nat. Labs., POB 5800 , Albuquerque, NM 87185, USA +1 (505) $8458659 / 8453115$ 30-Sept. 2 Fractals in Natural Sciences: Int. Conf. on the Complex Geometry of Nature

Budapest, Hungary M. Vicsek, MTA SZTAKI Comp. \& Autom. Res, Inst., POB 63, H-1518 Budapest 30 - Sept. 2 Optics of Excitons in Confined Systems: 3rd Int. Conf. Montpellier, France B. Gil, Case 074, Univ. de Montpellier, F-34095 Montpellier Cédex $5 \quad-/+33$ () 67143760

30 -Sept. 3 Active Galactic Nuclei Across the Electromagnetic Spectrum: IAU Symp. No. 159 Geneva, Switzerland T. J.-L. Courvoisier, Obs. de Genève, 51, ch. des Maillettes, $\mathrm{CH}-1290$ Sauverny
30 - Sept. 3 Thermophysical Props.: 13th European Conf. (ECTP-13) Lisbon, Portugal C.A. Nietro de Castro, Dept. de Quimica, Univ. de Lisboa, Campo Grande, Ed. C1 +351 (1) $7596305 / 7599404$ A: 31 Aug $93 /$ PP

30 - Sept. 3 Local Transport Studies in Fusion Plasmas: Int. Workshop Varenna, Italy G. Gorini, ISPP, via Celoria, 16, I-20133 Milano +39 (2) $2392637 / 2392205$ giuseppe @ imisiam.mi.cnr.it A: 10 Jun 93 / Ab: 10 Jun 93 / PP: 3 Sep 93 / 60 / LIT 500 000.-; incl. proc.

30 - Sept. 3 Induced Spectroscopy - Advances \& Applens.: NATO ARW Banff, Canada G.C. Tabisz, Physics Dept., Univ, of Manitoba, Winnipeg, Manitoba, R3T 2N2, Canada

30 - Sept. 4 Surface Science: 13th European Conf. (ECOSS-13) Warwick, UK C.F. McConville, Physics Dept., Univ. of Warwick, Coventry CV4 7AL, UK + 44 (203) $523353 / 692016$ ecos @ spec.warwick.ac.uk loP

30 - Sept. 4 Pion-Nuclear Physics: 5th Int. Oberjoch Meeting Oberjoch, Germany G.J. Wagner, Physik. Inst., Univ. Tübingen, Auf der Morgenstelle 14, W-7400 Túbingen physik1 @ dtuzdv5a

30 - Sept. 17 Materials Sci. \& Physics of Non-Conventional Energy Sources: Workshop Trieste, Italy ICTP, POB 586, 1-34100 Trieste + 39 (40) 22401 / 224163

31 - Sept. 3 Applens. of Synchrotron Radiation in Crystallography: 16 th IUCr Congress Sat. Meeting Beijing, PRC Y. Zhao, Beijing Synchrotron Rad. Fac., IHEP, POB 918, Beijing 100039, PRC $\quad-/+86$ (1) 18213374

31. Sept. 4 Accelerators in Applied Res. \& Technology: 3rd European Conf. Orléans, France J.-L. Debrun, Centre d'Etudes et de Recherches par Irradiation, CNRS, 3A, rue de la Ferollerie, F-45071 Orléans Cédex $2 \quad-1+33$ () 38630271

\section{SEPTEMBER}

Sept. Very Long Baseline Interferometry Technology - Prog. \& Future Obs. Possibilities: Conf. Kyoto, Japan M. Morimoto, Nobeyama Radio Obs., Nobeyama, Minimaki, Minamisaku, Nagano 384-13, Japan

Sept. Atmospheric Studies by Optical Methods: NATO ARW Apatity, Murmansk, Russia K. Henriksen, Auroral Univ., University of Tromse, $\mathrm{N}-9000$ Tromsø

Sept. IUPAP Gen. Ass. Nara, Japan J.S. Nilsson, IUPAP, Vasaparken Univ., S-411 24 Göteborg +46 (31) $631883 / 634660$

Sept./Oct. Austrian Physical Soc. Ann. Meeting Graz, Austria H. Jaeger, TU Graz, Petersgasse 16, A-8010 Graz NP/350

3-10 Acoustics: 14 th Int. Cong. Beijing, PRC Inst. of Acoustics, Chinese Acad. of Sciences, POB 2712, Beijing 100080 , PRC

3-12 Nonlinear Evolution Equations \& Dynamical Systems: 9th Workshop (NEEDS '93) Gallipoli, Italy M.C. Gerardi, Dip. di Fisica, Univ. di Lecce, via Arnesano, I-73100 Lecce +39 (832) 620467 ) 620505 gerardi @ lecce.infn.it A: 10 May 93/ PP / LIT $950000 . ;$ incl. board, lodge

5-9 Bubble Dynamics \& Interface Phenomena Birmingham, UK J.R. Blake, School of Mathematics \& Statistics, Univ. of Birmingham, Edgebaston, Birmingham B15 2TT, UK $\quad-/+44$ (21) 4143907 IUTAM

5-11 Electron Microscopy: 3rd Joint Meeting Zurich, Switzerland

5-11 Euroanalysis Edinburgh, UK

6-10 Thin Films: Int. Conf. (ICTF 9) Vienna, Austria A. Wagendristel, Inst. f. Angewandte und Tech. Physik, TU Wien, Wiedner Haupstrasse 8-10, A-1040 Vienna IUVSTA

6-10 Position-Sensitive Detectors: 3rd London Conf. London, UK D.C. Imrie, Physics Dept., Brunel Univ., Uxbridge, Middlesex UB8 3PH, UK +44 (895) $274000 / 272391 \quad$ imrie @ ph.brunel.ac.uk A: 30 Jul 93 / Ab: 23 Jun 93 / PP / £ 240.-; 150.- students; incl. proc.

6-10 Physical Chem. of Molecules \& Grains in Space: 50 th Internat. Meeting on Physical Chemistry Obernai, France Soc. Française de Chimie, 10, rue Vauquelin, F-75005 Paris $\quad-/+33$ (1) 43315945 6-10 Infrared \& Millimeter Waves: 18th Ann.SPIE Int. Conf. + Space Terahertz Technology: Symp. Colchester, UK K.J. Button, 2095 A-I-A, N. Indialantic, FL 32903, USA 
6-10 Radiation Effects in Insulators: 7th Int Conf. (REI-7) Nagoya, Japan C. Kusa-Ku, Physics Dept., Nagoya Univ., Nagoya 464-01, Japan

6-10 Amorphous Semiconductors - Science \& Technology: 15th Int. Conf. (ICAS-15) Cambridge, UK A.J. Snell, Elect. Engng. Dept., Edinburgh Univ. Edinburgh EH9 3JL, UK +44 (31) 6505650 6506554 a.j.snell @ ee.ed.ac.uk IUPAP 6-18 Excimer Lasers - The Tools, Fundamental Processes \& Applicns.: NATO ARW Aghia Pelaghia, Crete L.D. Laude, Dept. of Materials \& Processes, Univ. of Mons-Hainaut, Ave. Maistriau, 23, B-7000 Mons

7-10 Quantum Well Intersubband Transition Physics \& Devices: NATO ARW Whistler, Canada H.C. Liu, NRC, Inst. for Microstructural Sci., Ottawa, Canada, K1A OR6, Canada

8- 10 Nature \& Scope of Measurement Science London, UK IMEKO, POB 457, H-1317 Budapest +36 (1) $1531562 / 1561215$

10 - 13 European Quantum Electronics Conf. (EQEC '93) Florence, Italy M. Inguscio,

LESNL, largo E. Fermi, 2, 1-50125 Florence +39 (55) $229561 / 224072$

12-16 Optical Communication: 19th European Conf. (ECOC '93) Montreux, Switzerland R.P. Salathé, EPFL, CH-1051 Lausanne +41 (21) $6933338 / 6933701 \quad$ PP: 6 Apr 93

12-16 Applicns. of Density Functional Theory in Chemistry \& Physics: 5 th Int. Conf Como, Italy A. Gamba, Milano Univ., via Castelnuovo, 1-22100 Como +39 (31) $326219 / 326280 \quad \mathrm{PP} / 150 /$ LIT 250 000.- (300 000.- after deadline); 150000 . students ; incl. proc.

12-17 Solid State lonics: Int. Conf. (ISSIC-9) The Hague, The Netherlands A.M.C. Barrow, Lab. voor Toegpaste Anorganische Chemie, Julianalaan 136, NL-2628 BL Delft

13-15 Dynamics at the Gas-Solid Interface: Joint Meeting of the RSC, AICF, SCI, DBG Cambridge, UK Y.A. Fish, Royal Soc. of Chem., Burlington House, London W1V OBN, UK +44 (71) 4378656 / 7341227 DBG, SFC, AICF

13- 16 Radiations \& Their Effects on Devices \& Systems: 2nd European Conf. St. Malo, France Service Electronique, CEA-DAM, BP 12, F-91680 Bruyères Le Châtel

13-17 Structure, Interaction \& the States of Matter: 4th Brioni Int. Conf. on Interdisciplinary Topics Brijuni, Croatia S.D. Bosanac, Ruder Bošković Inst. Bijenicka 54, POB 1016, Croatia, 41001 Zagreb +38 (41) $435111 / 272648$ danko.bosanac @ irb.ac.mail.yu A: 15 May 93 / Ab: 15 May 93 / NP 100 / SUS 180.-; 50.- students; incl. board, lodge 13-17 Problems \& Methods in Mathematical Physics: 10 th Cont. Chemnitz, Germany L. Jentsch, Fach. Mathematik, TU Chemnitz, Postfach 964, O-9010 Chemnitz $\quad-/+49$ (71) 5612140 13-18 Magnetoelectric Interaction Phenomena in Crystals: 2nd Int. Conf. (MEIPIC-2) Ascona, Switzerland $\quad$ O. Hirth, Secr. de Chimie appliquée Univ. de Genève, 30 , quai $E$. Ansermet, $\mathrm{CH}-1211$ Geneva $4+41$ (22) $7026408 / 3296102$ meipic (1) sc2a.unige.ch ETHZ

14 - 17 European Phys. Soc. 9th Gen. Conf. Trends in Physics (EPS-9) Florence, Italy V. Tognetti, Dipt. di Fisica, Univ. di Firenze, largo E. Fermi, 2, I-50125 Florence +39 (55) $2298141 / 229330 \quad$ eps9 @ fi.infn.it A: 15 May $93 /$ Ab: 15 May $93 /$ PP / $1000 /$ SFR 450.-; 350.- nat. soc. mbrs; 300 .- IOM's; 120.- students (-15\% before 15 Jun 93$) \quad C E C$

14 - 17 Electron Microscopy \& Analysis Group of IOP (EMAG '93) Liverpool, UK Meetings Office, Inst. of Physics, 47 Belgrave Sq., London SW1X $8 Q X$, UK +44 (71) $2356111 / 2596002$

14-17 Non-Conventional Optical Imaging Elements: Int. Coll. Wroclaw, Poland J. Nowak Physics Inst., TU Wroclaw, Wybrzeze Wyspianskiego 27. PL-50-370 Wroclaw $\quad-/+48$ (71) 223664

14-17 Non-Conventional Optical Imaging Elements: Topical Meeting Rydzyna or Wroclaw. Czecho-Slovakia

147, F-91043 Orsay Cédex

15 - 17 FASE 10th Int. Symp. Bucharest, Romani Acc. Comm. of the Romanian Acad., Calea Victoriei No. 125 , Sector 1, R-71102 Bucharest $\quad+40(0)$ $502548 / 504794$
18-22 Liquid Matter: 2nd EPS Conference Florence, Italy M. Zoppi, CNR, Ist. di Elettronica Quantistica, via Panciatichi, 56/30, 1-50127 Florence +39(55) $416128 / 414612$ zoppi @ iffidg.fi.cnr.it A: 15 May 93 I Ab: 15 May 92 / PP / 500

18-23 Very High Resolution Spectroscopy: European Res. Conf. in Physics - France J. Hendeković, ERC, ESF, 1, q. Lezay Marnésia, F-67000 Strasbourg Cédex +33 () 88767135 / $88366987 \quad$ A: 15 May 93 / Ab: 15 May 93 / NP / 100; incl. board, lodge ESF, CEC

18-23 Electronic Structure of Solids: European Res. Conf. in Physics Thessaloniki, Greece J. Hendekovic, ERC, ESF, 1, quai Lezay Marnésia, F-67000 Strasbourg Cédex +33 () $88767135 / 88366987 \quad$ A: 15 May 93 / Ab: 15 May 93 / NP/ 100; incl. board, lodge ESF, CEC

19-23 Theory \& Phenomenology in Astroparticle \& Underground Physics: 3rd Int. Workshop (TAUP 93) Assergi, Italy Lab. Naz. del Gran Sasso 1-67010 Assergi +49 (862) $437231 / 410795$ 19-23 Physics \& Methods in Criticality: Topical Meeting Nashville, TN, USA M. Westfall, ORNL, Martin Marietta Energy Syst., POB 2008-6370, Oak Ridge, TN 37831-6370, USA

19-25 Adv. Quantum Field Theory \& Critical Phenomena: European Res. Conf. in Physics Ascona, Switzerland J. Hendeković, ERC, ESF, 1. quai Lezay Marnésia, F-67000 Strasbourg Cédex +33 () $88767135 / 88366987$ A: 15 May 93 I Ab: 15 May 93 / NP / 100; incl. board, lodge ESF, CEC

19-25 Phenomena in lonized Gases: 21st Int Conf. (ICPIG-21) Bochum, Germany U. Arend, Arbeitsgemeinschaft Plasmaphysik, Ruhr Univ., Postfach 102148, W-4630 Bochum $1 \quad-/+49$ (234) 7094178 Ab: 15 Jan 93 / PP: 15 May 93 / DM 480. IUPAP

20-22 Fractals, Chaos \& Predictibility in the Oceans: Chapman Conf. Galway, Ireland A.D. Kirwan, Dept. of Oceanography, Old Dominion Univ., Norfolk, VA 23529, USA

20-22 Nonlinear Guided Wave Phenomena Cambridge, UK OSA, Meetings Dept., 2010 Massachusetts Ave. NW, Washington, DC 20036, USA +1 (202) $2238130 / 2231096$

20-23 Polish Phys. Soc. Gen. Meeting Cracow, Poland M. Kisiel, Inst. of Physics, Jagellonian Univ. ul. Reymonta 4, PL-30-059 Cracow

$336377 / 337086$ ufkisiel @ plkrcy11 $20-24$ Solar Coronal Structures: IAU Coll. No. 144 Tatranská Lomnica, Czecho-Slovakia V. Rusin Astronom. Inst., Slovak Acad. of Sciences, CS-059 60 Tatranská Lomnica

20 - 24 Capture Gamma-Ray Spectroscopy \& Related Topics: 8 th Int. Symp. Fribourg, Switzerland J. Kern, Physics Dept, Univ. Fribourg, $\mathrm{CH}-1700$ Fribourg $+41(37) 826233 / 826519 \quad$ kern @ cfruni52 Ab: 31 Mar 93/PP

20-24 Textures of Materials: 10 th Int. Conf. (ICO TOM 10) Clausthal, Germany Inst. F. Metallkunde, Grosser Bruch 23, W-3392 Clausthal-Zellerfeld +49 (5323) $722244 / 722340$

20-24 Emerging Nuclear Energy Systems: 7 th Int. Conf. (ICENES '93) Makuhari, Japan T. Hiraoka, Dept. of Reactor Engng., JAERI, Tokaimura, Ibaraki-ken, 319-11, Japan +81 (292) $825517 / 826122$

20-25 IUPAP 21st Gen. Ass.

Nara, Japan J.S. Nilsson, IUPAP, Vasaparken Univ., S-411 24 Göteborg +46 (31) $631883 / 634660$

20-Oct. 1 Composite Media \& Homogenization 2nd Workshop Trieste, Italy ICTP, POB 586, 1-34100 Trieste $+39(40) 22401 / 224163$

21- 22 Physical Acoustics \& Ultrasonics - Ann Rev.: IoP Physical Acoustics Group Sheffield, UK Inst. of Physics, 47 Belgrave Sq., London SW1X $8 Q X$. UK +44 (71) $2356111 / 2596002$

$21-23$ Potentials \& Correlations in the Physics of Cond. Matter: A Symp. in Honour of M.P. Tos Pavia, Italy G. Senatore, Dipt. di Fisica Teorica, Trieste Univ., Strada Costiera, 11, I-34014 MiramareGrignano+39 (40) $2240278 / 224701$ mh9ts1byx @ icineca2 A: 30 Jun 92 / Ab: 31 May 92 / 31 Oct 92 / P: 31 Oct 92
22 - 24 Computer Aided Engineering Education: Conf. Bucharest, Romania D. loan, Polytechnic Inst., Spl. Independentei 313, R-77206 Bucharest +40 (1) $3121190 / 3121190$

25 - 30 Quantum Optics: European Res. Conf. in Physics Davos, Switzerland J. Hendekovic, ERC, ESF, 1, quai Lezay Marnésia, F-67000 Srasbourg Cédex +33 () 88767135 / 88366987 A: 15 May 93/Ab: 15 May 93 / NP/ 100; incl. board, lodge ESF, CEC

25 - 30 Dynamical Props. of Solids: European Res. Conf. in Physics Lunteren, nr. Arnhem, The Netherlands J. Hendekovic, ERC, ESF, 1. quai Lezay Marnésia, F-67000 Srasbourg Cédex +33 () $88767135 / 88366987$ A: 15 May $93 /$ Ab: 15 May 93 / NP / 100; incl. board, lodge ESF, CEC

26 - 30 Structural Intermetallics: Int. Symp. (ISSI) Champion, PA, USA R. Darolia, GE Aircraft Engines, Mail Drop M-89, Cincinnati, OH 45215, USA +1 (513) $2434509 / 2433250$ PP: 1 Feb 93 MMMS

27 - Oct. 1 Transitions in Oligomer and Polymer Systems: Europhysics Conf. on Macromolecular Physics UIm, Germany H.G. Kilian, Univ. of UIm, Experimentelle Physik, Albert-Einstein-Allee 11, W-7900 Ulm +49 (731) $5023010 / 5023036 \quad$ Ab: 31 May $93 /$ PP: 30 Nov 93 / SFR 400.-; 350.- IOM's, 100.students (before $15 \mathrm{Jul} \mathrm{93);} \mathrm{incl.} \mathrm{proc.}$

27 -Oct. 1 Fusion Reactor Materials: Int Conf. (ICFRM-6) Stresa, Italy P. Schiller, Inst. for Adv. Materials, JRC, I-21020 Ispra +39 (332) $789988 / 789434$

27 - Oct. 1 Accelerator Mass Spectrometry: 6th Int. Conf. (AMS-6) Canberra, Australia GPO Box 2200, Canberra, ACT 2601, Australia +256 (6) $2498105 / 2573256$

27 - Oct. 22 Telematics: Workshop Trieste, Italy ICTP, POB 586, 1-34100 Trieste +39 (40) 22401 I 224163

29 -Oct. 1 Soft Magnetic Materials: 11th Int. Conf. (SMM11) Venice, Italy F. Fiorillo, IENGF, corso M. d'Azeglio, 42, I-10125 Turin +39 (11) $3488933 / 6507611 \quad$ smm11 @ ien10.to.cnr.it A: 15 Jun 93 / Ab: 31 Mar 93 / PP: 31 Jul 93/200/ LIT 550 000.-; 450 000.- IOM's; 100 000.- students; incl. proc.

\section{OCTOBER}

Oct. Italian Phys. Soc. 79th Ann. Cong. Udine, Italy IPS, via L. degli Andalo, 2, 1-40124 Bologna +39 (51) $331554 / 581340 \quad$ Ab: 31 May 93 / 800 / Italian / LIT 120 000.-; 70 000.- mbrs.

3-6 Japan Phys. Soc. 1st Sectional Meeting Kochi-shi, Japan PSJ, Kikai-Shinko Building, 3-5-8 Shiba-Koen, Minato-ku, Tokyo 105, Japan +81 (3) $34342671 / 34320997$

3-8 Interdisciplinary Laser Sci. Conf.

Toronto, Canada OSA, Meetings Dept., 2010 Massachusetts Ave. NW, Washington, DC 20036, USA +1 (202) $2238130 / 2231096$

4-7 Fusion Energy: Symp. Burlington, VT, USA A.M. Dawson, 175 Albany St., Cambridge, MA 02139-4307, USA +1 (617) $2535547 /$. 4-8 Applicns. of Surface \& Interface Analysis: 5th Conf. (ECASIA '93) Catania, Italy Consorzio Catania Ricerche, V.le A. Doria, 6, I-95125 Catania +39 (95) $221635 / 339734$ PP $/ 400$ CNR, ENEA

5- 7 Lasers, Optics \& Electro-Optics: Int. Conf. Brighton, UK Sira Communications Ltd., South Hill, Chiselhurst, Kent, BR7 5EH, UK $4672636 / 4677258$

11-13 Nanometre-Scale Methods in X-Ray Technology: Europhysics Industrial Workshop (EIW-9) Veldhoven, The Netherlands D.G.K. de Boer, Philips Res. Labs, Postbus 80.000, NL-5600 JA Eindhoven $\quad-/+31$ (40) 744675 A: 1 Aug $93 /$ NP $<50 /$ SFR 750.(500.- EPS mbrs.); incl. board, lodge

12 - 15 Japan Phys. Soc. 2nd Sectional Meeting Okayama-shi, Japan PSJ, Kikai-Shinko Building, 3-5-8 Shiba-Koen, Minato-ku, Tokyo 105, Japan +81 (3) $34342671 / 34320997$ 
18 - 22 Accelerator \& Large Experimental Physics Control Systems: Int. Conf. (ICALEPCS '93) Berlin, Germany

W. Busse, HMI, Postfach 390128, W-1000 Berlin $39+49(30)$ $80092415 / 80092097$ busse @ vax.hmi.dbp.de PP / 350 / DM 450.-; 400.- IOM's; 150.- students; incl. proc.

20-22 Gradient Index Optical Systems: 4th Micro-Optics Conf. + 11th Topical Meeting (MOC/ GRIN'93) Kawasaki, Japan Y. Kokubun, Div. of Electrical \& Computer Engng., Yokohama Nat. Univ. Tokiwadai, Hodogayaku, Yokohama 240, Japan +81 (45) $3351451 / 3381158$

31-Nov. 4 Computation of Electromagnetic Fields: 9th COMPUMAG Conf. Miami, FL, USA Dept. of Electrical \& Computer Engng., Florida Int. Univ., Miami, FL 33199, USA +1 (305) 3483711 3483707 ulerf @ servax.fiu.edu

\section{NOVEMBER}

Nov. Physique'93 Paris, France C. Sébenne, 33, rue Croulebarbe, F-75013 Paris $+33(1)$ $47073298 / 43317426$

1-3 Numerical Simulation of Nonisothermal Flow of Viscoelastic Fluids Kerkrade, The Netherlands J.F. Dijksman, KIvl, Postbus 30424 , NL-2500 GK The Hague $\quad-/+31$ (70) 3919840 1-4 IEEE Ultrasonics Symp. Baltimore, MD USA H.J. Salvo, Westinghouse Electronic Systems Group, 333 Gordon Avenue, Severna Park, MD 21146, USA

1-5 American Phys. Soc. Plasma Physics Div. Fall Meeting St. Louis, MO, USA APS, 335 E. 45th St., New York, NY 10017, USA $+1(212)$ $6827341 / 6872532$

2- 5 Nuclear Science Symp. San Francisco CA, USA E.J. Lampo, Lawerence Berkeley Lab. 1 Cyclotron Road, 29/10, Berkeley, CA 94720, USA +1 (415) $4866779 / 4864122$

8-12 High-Energy Physics: Trieste Conf. (tentative) Trieste, Italy ICTP, POB 586, I-34100 Trieste $\quad+9(40) 22401 / 224163$

8 - 26 Non-Linear Dynamics \& Earthquake Pre diction: 2nd Workshop Trieste, Italy ICTP, POB 586, I-34100 Trieste+39 (40) $22401 / 224163$ 10- 12 Temperature \& Thermal Measurement in Ind. \& Sci.: 5 th Int. Symp. (TEMPMEKO-5) Prague, Czecho-Slovakia J. Krai, TECH-MARKET, POB 44 , CS-160 17 Prague $6 \quad-/+42$ (2) 355184

10-13 Swedish Phys. Soc. "Physics Days" Biennial Meeting Umeá, Sweden C. Ekstrom The Svedberg Lab., Uppsala University, POB 533 S-75121 Uppsala +46(18) $183112 / 183833$ 500

14- 19 American Nuclear Society Winter Meeting San Francisco, CA, USA ANS, 555 N. Kensington Ave., La Grange Park, IL 60525, USA $3526611 / 3526464$

14-19 Optical Society of America Ann. Meeting (OPTCON '93) San Jose, CA, USA OSA, Meetings Dept., 2010 Massachusetts Ave. NW, Washington, DC 20036, USA

2231096

15-18 Magnetism \& Magnetic Materials: 37th Conf. (MMM-37) Minneapolis, MN, USA IEEE Inc. Conf. Cordination, 345 E 47th St., New York, NY 10017, USA

19 Measurement on Optical Devices London, UK IEE Conf. Services, Savoy Place, London WC2R OBL, UK, London WC2R OBL IOP

22 - Dec. 3 VLSI Technology: Workshop Trieste, Italy ICTP, POB 586, I-34100 Trieste +39(40) $22401 / 224163$

29 -Dec. 3 Materials Res. Society Fall Meeting Boston, MA, USA M.N. Geil, MRS, 9800 McKnight Rd., Pittsburg, PA 15237, USA +1 (412) $3673003 /$ Ab: 1 Jul $93 / P P$

\section{DECEMBER}

Dec. 12 - 18 Methods \& Procedures in Mathematical Physics Freiburg im Breisgau, Germany Math Forschungsinst., Albertstrasse 24, W-7800 Freiburg linv.

15- 17 Potential Energy Surfaces \& Organic Reaction Paths: Faraday Symp. 29 London, UK Y.A. Fish, Royal Soc. of Chem., Burlington House, London W1V OBN, UK
20 - 22 Cond. Matter \& Materials Physics: Div. Ann. Conf. Leeds, UK Cong. \& Meetings Office, Inst. of Physics, 47 Belgrave Sq. London SW1X $8 Q X$, UK +44 (71) $2356111 / 2596002$

\section{Conferences 1994}

1994 Adv. Toroidal Devices with Innovative Physics \& Technology Concepts Varenna, Italy ISPP, Via Celoria, 16, 1-20133 Milano

1994 Self Organisation of Biopolymers \& Macromolecules: Joint Meeting und Bunsen-Diskussionstagung Y.A. Fish, Royal Soc. of Chem. Burlington House, London W1V OBN, UK

1994 DAGA: 20. Tagung Dresden, Germany Lenk, TU Dresden, Mommsenstr. 13, 0-8027 Dresden 1994 European Laser Assocn. 7th Biennial Cong. Barcelona, Spain Southampton Lase Unit, Royal South Hants Hospital, Southampton SO9 4PE, UK

1994 Solid Compounds of Transition Elements 11 th Int. Conf. Wroclaw, Poland W. Suski Low Temp. \& Structure Res., POB 937, PL-50-950 Wroclaw 2

1994 Atomic \& Molecular Physics of Ionised Gases: 12th European Sectional Conference (12th ESCAMPIG)

$\mathrm{CH}-1213$ Petit-Lancy 2

1994 Controlled Fusion \& Plasma Physics: 21th European Conf. Marseilles, France F.W. Sluijter, Appl. Phys. Dept., Eindhoven Univ. of Tech., Postbus 513, NL-5600 MB Eindhoven

1994 or 1995 Polarization Phenomena in Nuclear Physics: 8th Int. Conf. + High-Energy Spin Physics: 10th Int. Conf. Bloomington, IN, USA J. Arvieux, Lab. Nat. Saturne, CEN Saclay, F-91191 Gif-sur-Yvette Cédex

Jan. 11-15 American Astro. Soc. 183rd Meeting Arlington, VA, USA AAS, Suite 300,2000 Florida Ave NW, Washington, DC 20009, USA

- Feb. Deep Inelastic Scattering \& Related Physics Eilat, Israel A. Levy, School of Physics, Tel-Aviv Univ., IL- 69978 Tel Aviv

21-25 Fibre Optic Sensing \& Environmental Monitoring: 2nd Int. Symp. \& Exhibition Prague, Czecho-Slovakia European Optical Soc., BP 147. F-91043 Orsay Cédex

21-25 Optical Fiber Communications Conf. (OFC '94) San Jose, CA, USA OSA, Meetings Dept., 2010 Massachusetts Ave. NW, Washington DC 20036, USA

Spring Positron Annihilation: 10th Ann. Conf. (ICPA-10) Beijing, PRC B. Cao, Physics Dept. Tsinghua Univ., Beijing 100084, PRC

Spring Infrared Lasers: Topical Conf. (CIRP-6: 6th Int. Conf. on Infrared Physics) Ascona, Switzerland F.K. Kneubūhl, Infrared Physics Lab., ETH, HPF Hönggerberg, $\mathrm{CH}-8093$ Zurich

March Spatio-Temporal Chaos \& Cellular Structures in Thermal Processes \& Convection

Grenoble, France H. Peerhossaini, ISITEM La Chantrerie, CP 3023 , F-44087 Nantes Cédex

$14-18$ 58. Physikertagung der DPG Hamburg. Germany

W-5340 Bad Honnef 1

28 - 31 (to be confirmed) Phys. Society of Japan 49th Ann. Meeting Fukuoka-shi, Japan PSJ, KikaiShinko Building, 3-5-8 Shiba-Koen, Minato-ku, Tokyo 105, Japan

21- 25 Frühjahrstagung der Arbeitskreis Festkörperphysik bei der DPG Münster, Germany German Phys. Soc., Haupstr. 5, W-5340 Bad Honnef 1 28 - April 1 European Phys. Soc. Cond. Matter Div. 14th Gen. Conf. Madrid, Spain E. Thomas, EPS, POB 69, $\mathrm{CH}-1213$ Petit-Lancy 2

April/May Computing in High-Energy Physics (CHEP'94) San Francisco, CA, USA S. Loken, Fermilab

April 1-2 Radiation Protection \& Management Theory \& Practice Oxford, UK D.N.S. Dixon, Soc. for Radiological Protection, 67 Oatlands Park Linlithgow, West Lothian EH49 6A5, UK

4-9 High Power Lasers: 1st Int. Symp.

Vienna, Austria European Optical Soc., BP 147. F-91043 Orsay Cédex

6-8 The Structure \& Dynamics of van der Waals Molecules: Disc. Meeting

Y.A. Fish, Royal Soc. of Chem., Burlington House London W1V OBN, UK
8 The Netherlands' Phys. Soc. Spring Meeting Utrecht, The Netherlands M. Hoogenboom, NNV, Julianalaan 74, NL-3722 GS Bilthoven

11- 14 Adv. in Reactor Physics: Topical Meeting Knoxville, TN, USA B.A. Worley, Martin Marietta Energy Syst., ORNL, POB 2008-6363, Oak Ridge, TN 37831-6363, USA

11- 15 Integrated Optics: Int. Symp. Lindau, Germany European Optical Soc., BP 147 F-91043 Orsay Cédex

$11-15$ Materials Res. Soc. Spring Meeting San Francisco, CA, USA

9800 McKnight Rd., Pittsburg, PA 15237, USA

17 - 22 Space Optics: Int. Symp. (ICSO'2)

Garmisch, Germany European Optical Soc.

BP 147, F-91043 Orsay Cédex

18- 22 American Phys. Soc. Spring Meeting

Washington, DC, USA

- May Computer Coupling of Phase Diagrams \& Thermochemistry: 23rd Ann. Int. Cont. (CALPHAD-23) Madison, WI, USA L. Kaufman, 21 Erie St., Cambridge, MA 02139, USA

May Digital Holography/Diffractive Optics: Topical Meeting Prague, Czecho-Slovakia European Optical Soc., BP 147, F-91043 Orsay Cédex

8-13 Quantum Electronics \& Laser Sci. Conf./ Conf. on Lasers \& Laser Optics (CLEO/QELS '94) Anaheim, CA, OSA, Meetings Dept. 2010 Massachusetts Avenue, NW, Washington, DC 20036, USA

12 - 14 DBG f. Physikalische Chemie Hauptversammlung 1994 Berlin, Germany Bunsen-Ges. f. Physikalische Chemie.

W-6000 Frankfurt-am-Main 90

24-27 Assessing Impact of Nuclear Facilities on Human Health \& the Environment: Topical Meeting Arlington, TX, USA 400 N. Olive, LB-81, Dallas, TX 75201, USA

25 - June 6 Int. Optometric \& Optical League Ann. Conf. -, Canada D. Leason, IOOL, 10 Knaresborough PI., London SW5 OTG, UK

June 6-8 Plasma Sciences: Conf. Santa Fe, NM, USA A.L. Perrat, Los Alamos Nat. Lab., Group X-10, MS B-259, POB 1663, Los Alamos, NM 87545 USA

6 - 10 Optical Interference Coatings: Int. Symp. Grenoble, France European Optical Soc., BP 147 F-91043 Orsay Cédex

6-10 8th Quadrennial STP Symp. Sendai, Japan H. Oya, JPL, Caltech, 4800 Oak Grove Drive, Pasadena, CA 91109-8099, USA

12-15 Canadian Assocn. of Physicists Cong. Regina, Canada F.M. Brûlé, Suite 903, 151 Slater St. Ottawa Ontario K1P $5 \mathrm{H} 3$, Canada

19-24 American Nuclear Society Ann. Meeting New Orleans, LO, USA ANS, 555 N. Kensington Ave., La Grange Park, IL 60525, US

20-23 Advanced Materials in Optics, ElectroOptics \& Communication Technologies: 8th CIMTEC Conf. Florence, Italy European Optical Soc., BP 147, F-91043 Orsay Cédex

20-23 Magnetism \& Magnetic Materials: Int. Joint Conf. (MMM/INTERMAG-6) Albuquerque, NM, USA Courtesy Associates, Inc., 65515 th St. NW, Suite 300 , Washington D.C. 20005, USA

20 - 23 24th AIAA Fluid Dynamics, Plasmadynamics \& Laser Conf. Colorado Springs, CO, USA AIAA, Meetings Dept., 370 L'Enfant Promenade SW, Washington, DC 20024, USA

21-23 Applicns. of Photonic Technology: Int. Conf. Toronto, Canada G. Lampropoulos, 560 Lauder Ave., Toronto, Ontario, M6E 3J6, Canada 27 - July 1 4th European Particle Accelerator Conf. (EPAC94) London, UK Ch. Petit-JeanGenaz, CERN-AC, $\mathrm{CH}-1211$ Genève 23

27 - July 1 Precision Elecromagnetic Measurements: Conf. (CPEM '94) Boulder, CO, USA D.B. Sullivan, Time \& Freq. Div., NIST, Boulder, CO 80303 , USA

Summer Norwegian Phys. Soc. Physics Meeting Bergen, Norway G. Jarret, Physics Dept., Inst. for Energy Technology, Postboks 40, N-2007 Kjeller

- July 4-9 Materials \& Mechanisms of Superconductivity - High-Temperature Superconductors (M2S-HTSC) Grenoble; France M. Cyrot, Lab. Louis Néel, 25, ave. des Martyrs,

F-38042 Grenoble Cédex

10-22 COSPAR 30th Plenary Meeting \& Ass. Activities Hamburg, Germany Committee on Space Res., 51 blvd. de Montmorency, F-75006 Paris 
17-21 Ultrasound: World Congress Sapporo Japan M. Jukuda, Div. of Ultrasound, Sapporo Med Coll., W-16, Chu-ku, Sapporo, Japan

17-22 Electron Microscopy: 13th Int. Congress (ICEM-13) Paris, France Soc. Française de Microscopie Electronique, 67, rue Maurice-Gunsbourg F-94205 Ivry-sur-Seine Cédex

18-23 Mathematical Physics: 11th Int. Conf (IAMP-11) Paris, France D. lagolnitzer Physique Théorique, Centre d'Etudes de Saclay, CEA, F-91191 Gif-sur-Yvette Cédex

21 - 27 High-Energy Physics: 27th Int. Conference Glasgow, UK Cong. \& Meetings Office, Inst. of Physics, 47 Belgrave Sq. London SW1X 8QX, UK 31-Aug. 5 Atomic Physics: 14th Int. Conf. (ICAP 14) Boulder, CO, USA C.E. Wieman, JILA Univ, of Colorado, Boulder, CO 80309 , USA

- Aug. 14-Sept. 2 Int. Astronomical Union Triennia Gen. Ass. The Hague, The Netherlands P. Wittebo Congrex, Keizersgracht 782, NL-1017 EC Amsterdam 20-22 Int. Geoscience \& Remote Sensing Symp. (IGARSS '94) Pasadena, CA, USA OSA, Meetings Dept., 2010 Massachusetts Ave. NW Washington, DC 20036, USA

22- 25 Optical Compting: Int. Meeting (OC '94) Edinburgh, UK

B.S. Wherrett, Physics Dept. Heriot-Watt Univ., Edinburgh EH14 4AS, UK

22- 26 Physics Computing '94: 6th Joint EPS APS Int. Conference on Physics Computing (PC'94) Manno, TI, Switzerland

R. Gruber, CSCS via Cantonale, $\mathrm{CH}-6928$ Manno

22-26 Magnetism: Int. Conf. (ICM 94) Poznan Poland J. Morkowski, Inst. of Molecular Physics, Polish Acad. of Sci., Smoluchowskieg 17/19,

PL-60-179 Poznan

28 - Sept. 2 15th European Crystallographic Meeting (ECM-15) Leipzig, Germany P.Paufler, Inst. f. Kristallographie, Univ. Leipzig, Scharnhorststr 20, O-7030 Leipzig

- Sept. Balkan Physical Union 2nd Gen. Cont. (BPU-2) Izmir, Turkey K.G. Akdeniz, Physics Dept., Istanbul Univ, TR-34459 Vezneciler

Sept. Portuguese Phys. Soc. "Fisica '94" Biennial General Meeting -, Portugal C.M. Ferreira Centro de Electrodinamica, Univ. Técnica, Inst. Superior Técnico, P-1096 Lisbon Codex

5 - 9 IMEKO 13th World Cong

IMEKO, POB 457, $\mathrm{H}-1371$ Budapest 5

11-16 Molecular Spectroscopy: 22nd European Cong. (EUCMOS-22)

B. Schrader, Universitätsstr., W-4300 Essen 1

11-16 ESSDERC 1994 Edinburgh, UK

Cong. \& Meetings Office, Inst. of Physics, $47 \mathrm{Bel}$ grave Sq., London SW 1 X $8 Q X$, UK

21-26 Fundamental Physical Aspects of the Strength of Crystalline Materials: 2nd Int. Conf. (JMIC-2) Sendai, Japan H. Oikawa, Dept. of Sci., Tohoku Univ., Sendai 980, Japan

Oct. Plasma Physics \& Controlled Fusion

Res.: 15th Int. Conf. Moscow, Russia IAEA,

Conf. Service Sect., POB 100, A-1400 Vienna

581340

Oct. Italian Phys. Soc., 79th Nat. Cong.

IPS,

via L. degli Andalo, 2, I-40124 Bologna

2-6 European Nuclear Cong. (ENC '94) Lyon, France ENS, POB 5032, CH-3001 Bern

30-Nov. 4 Optical Soc. of America Ann. Meeting (OPTCON '94) Boston, MA, USA OSA, Meetings Dept., 2010 Massachusetts Ave. NW, Washington, DC 20036, USA

- Nov.2-5 Ultrasonics: IEEE Symp. Cannes, France G.J. Quinten, Univ. de Paris, GPS, Tour 23 2. place Jussieu, F-75251 Paris Cédex 05

7-11 American Phys. Soc. Plasma Physics Div. Meeting Minneapolis, MN, USA APS, $335 \mathrm{E}$ 45th St., New York, NY 10017, USA

20-22 Cond. Matter \& Materials Physics Div Ann. Conf. Warwick, UK Cong. \& Meetings Office, Inst. of Physics, 47 Belgrave Sq., London SW1X 8QX, UK

\section{Schools 1993}

Jan. 18 - Feb. 5 Physics \& Technology of Lasers \& Optical Fibres: 4th Training College Trieste, Italy A. Denardo, ICTP, POB 586, 1-34100 Trieste +39 (40) $22401 / 224163 \quad$ A: 31 Jul $92 / \mathrm{lim}$
20 - Feb. 2 Ocean Processes in Climate Dynamics - Global \& Mediterranean Examples: Int.School of Climatology (6th Course)

Erice, italy

P. Malanotte-Rizzoli, MIT, Rm, 54-1416, Cambridge, MA 02139, USA +1(617) $2532451 / 2536208$ 25 - Feb. 6 High-Energy Theory: St. Petersburg Winter School St. Petersburg, Russia St. Peters burg Nuclear Physics Inst., Gatchina, CIS-188350 St Petersburg +7(812) $2979196 / 37196$ osoffe @ Inpi.spb.su A: 1 Dec 92 / PP / \$ US 600.-; incl. board, lodge

31 - Feb. 3 Medium-Energy Physics with the 0 Facility at COSY Bad Honnef, Germany German Phys. Soc., Haupstr. 5, W-5340 Bad Honnef $1+49$ 2224) $71061 / 71063$

- Feb. 1-6 Fiber Optic Communication Technoogy, Systems \& Networks Garmisch Partenkirchen, Germany T. Persson, CEI-Elsevier/Europe, POB 910, S-612 01 Finspong +46(122) 17550 14347

Theo. \& Experimental Radiopropagation Physics: 3rd ICTP-URSI College Trieste, Italy ICTP, POB 586, I-34100 Trieste +39 (40) 22401 224163

8- 26 Optics: Winter College Trieste, Italy ICTP, POB 586, 1-34100 Trieste + 39 (40) 22401 $224163 \quad$ A: 31 Jul $92 /$ no fee

14-April 2 Neutrons \& Synchrotron Radiation for Condensed Matter Studies (HERCULES)

Grenoble, France M.C. Simpson, Secrétariat HER CULES, CNRS, BP 166, F-38042 Grenoble Cédex +33 () $76887986 / 76887981 \quad$ / A: 15 Oct 92 15- 27 The Physics of Phonons: 29th Winter School of Theo. Physics Karpacz, Poland T. Paszkiewicz, Inst. of Theo. Physics, University of Wroclaw, PI. Maxa Borna 9, PL-50-204 Wroclaw +48 (71) $201427 / 401467 \quad$ tapasz @ plwrtu11 A: 15 Jan 93 / PP / 100 / SUS 270.-; incl. board, lodge 15-27 Instrumentation in Particle Physics ICFA School Bombay, India M. Sheaf, Univ. of Wisconsin, Madison, USA sheaff @ wishep.earn 21-27 Collider Physics: Lake Louise Winter Inst. Lake Louise, Alberta, Canada A. Schaap man, Lake Louise Winter Inst., Physics Dept., Univ. f Alberta, Edmonton, Alberta T6G 2J1, Canad +1 (403) $4921052 / 4923408$ A: 11 Dec $92 /$ PP $<100$ / \$CDN 150.-; 50.- students; incl. proc.

24 - March 5 Structure of Matter as Revealed with Electroweak Probes: 32nd Int. Nuclear \& Particle Physics Univ. Meeting Schladming, Austria W. Plessas, Inst. f. Theo. Physik, Universitătsplatz 5 , A-8010 Graz +43 (316) $3805225 / 384091$ utp @ edvz.uni-graz.ada.at

$150 /$ inv.

28 - March 5 Physiik der Polymere: DPG-Schüle $f$ Physik (Kurs I) Bad Honnef, Germany German Phys. Soc., Haupstr. 5, W-5340 Bad Honnef $1+49$ (2224) $71061 / 71063$

Spring Multiphase Flow \& Heat Transfer Bases, Modelling \& Applicns.: Short Courses Zurich Switzerland G. Yadigaroglu, Inst. f. Energietechnik ETH-Zentrum, $\mathrm{CH}-8092$ Zurich +41 (1) 2564615 2622158

March 1-26 Computer Networks: 3rd College Trieste, Italy ICTP. POB 586, I-34100 Trieste +39 (40) $22401 / 224163$

6-13 Fullerenes \& Related Compounds: nt. Winterschool on Electronic Props. of Novel Materials Kirchberg Austria $\quad H$. Kuzmany, Inst. f Festkörperphysik, Universität Wien, Strudlhofgasse 4 A-1090 Vienna $\quad+43$ (1) $342630245 / 3103888$ 100

10 - 12 RF \& Microwave Component Modeling Davos, Switzerland T. Persson, CEI-Elsevier/ Europe, POB 910, S-612 01 Finspong $17550 / 14347$

15-19 Intro. to Polymer Sci.: Residential Course Bristol, UK S.M. Pringle, Dept. of Continuing Education, Wills Memorial Bldg. Queens Rd. Bristol, BS8 1HR, UK +44 (272) $303611 / 254975$ A: 26 Feb $93 /$ limited / £ 880 .

22-28 X-Ray Structure Analysis of Small \& Medium-Sized Molecules: 4 th Intensive Course Birmingham, UK D.J. Martin, Chem. Crystallography Lab., 9 Parks Rd., Oxford OX13PD, UK 29 - April 2 Colloid Science: Spring School Bristol, UK S.M. Pringle, Dept. of Continuing Education, Wills Memorial Bldg., Queens Rd., Bristol BS8 1HR, UK + 44 (272) $303611 / 254975$
April 28-30 Safety in Tritium Handling Technology: EUROCOURSE Ispra, Italy Eurocourses, JRC, I-21020 Ispra +39 (332) $787919 / 789839$ 29 - May 5 RF Engineering for Particle Accelerators: CERN Accelerator School Anacapri, Italy S. von Wartburg, SL Div., CERN, CH-1211 Geneva 23 castf@ cernvm.cern.ch +41 (22) 7824836

A. 1 Feb 93 / inv. / LIT 1400000

May 1-14 Quantum Optics: 2nd Erasmus School Heraklion, Crete, Greece P. Lambropoulos, FORTH-IESL, POB 1527, GR-711 10 Heraklion

3-7 Quality of Environmental Measurements EUROCOURSE Ispra, Italy Eurocourses, JRC I-21020 Ispra $\quad+39(332) 787919 / 789839$

9-26 Lasers \& Applications: 2nd EPS
Southern European School Elounda, Crete,
Greece D. Charalambidis, FORTH-IESL,
POB 1527, GR- 71110 Heraklion $+30(81)$
$210035 / 239735 \quad$ chara @ grearn
A: 15 Jan $93 /$ inv. $/<40$

17-29 Materials \& Crystallographic Aspects of High-T ${ }_{c}$ Superconductivity: Int. School of Crystallography (20th Course) Erice, Italy L. Riva di Sanseverino, Int. School of Crystallography, Piazza Porta San Donato, 1, 1-40126 Bologna +39 (51) 243556 I $243336 \quad$ t54bom12 @ icineca A: 30 Nov 92 । SFR 1800.-; incl. board, lodge

17 - June 11 Plasma Physics: Spring College Trieste, Italy ICTP, POB 586, I-34100 Trieste +39 (40) $22401 / 224163 \quad$ A: 31 Dec 92 17 - June 11 Computational Physics: College Trieste, Italy ICTP, POB 586, I-34100 Trieste +39 (40) $22401 / 224163 \quad$ A: 30 Nov 92

-June 1-9 Advances in Integrated Optics: Int. School of Quantum Electronics (18th Course) Erice, Italy M. Bertolotti, Dipt. di Energetica, Univ. degli Studi, "La Sapienza", via A. Scarpa, 14-16 +39 (6) 425787 / $4270183 \quad$ A: 1 Mar 93/PP / 75 / SUS $800 .-;$ incl. board, lodge; incl. proc.

7-9 Neural Networks \& Pattern Recognition Stockholm, Sweden Europe, POB 910, S-612 01 Finspong +46 (122) $17550 / 14347$

9-12 Dynamics Days Poznan Poznan, Poland G. Eilenberger, IFF der KFA Jülich, Postfach 1913 , W-5170 Jülich +49 (2461) $614073 / 612410$ iff042 @ djukfa11 A: 30 Apr 93/ Ab: $30 \mathrm{Apr} 93 /$ NP / $250 /=$ DM $350 .-;$ incl. board, lodge

14 - Aug. 13 High-Energy Physics: Summer School + Non-Accelerator Particle Physics: 3rd School

Trieste, Italy

+39 (40) $22401 / 224163$

$16-30 \quad$ Nonlinear Spectroscopy of Solids Adv. \& Applicns.: Int. School of Atomic \& Molecular Spectroscopy (11th Course) Erice, Italy B. di Bartolo, Physics Dept., Boston College, Chestnut Hill, MA 02167, USA +1 (617) 5523601 / 5528478 A: 3 Apr $93 /$ PP / SUS 1100.-; incl. board, lodge; incl. proc. NATO

20 - July 7 High-Energy Heavy lon Physics: Sum mer School Lake Balaton, Hungary J. Zimányi, KFKI Inst. for Particle \& Nuclear Research, POB 49 , $\mathrm{H}-1525$ Budapest

21-25 The Radon Issue: Int. Training Course Stockholm, Sweden M. Olast, DG XII-D-3, CEC R. de la Loi, 200, B-1049 Brussels +32 (2) 2350723 / 21 - July 7 Phenomenology \& Lattice QCD: Uehling Summer School Seattle, WA, USA S. Sharpe Physics Dept., University of Washington, Seattle, WA 98195, USA $\quad-1+1$ (206) 6850635

22 - July 2 Diagnostic Tools in Atmospheric Physics: Int. School of Physics "Enrico Fermi"-1 st Course Varenna, Italy G. Visconti, Dip. di Fisica, Univ dell'Aquila, via Vetoio, I-67010 Coppito $\quad+39(862)$ 433075 / $433033 \quad$ A: 1 May 93 / PP / 50 LIT $1700000 .-;$ incl. board, lodge SIF 28 - July 30 Computational Fluid Dynamics: Summer School Les Houches, France J ZinnJustin, Ecole de Physique Théorique, Côte de Chavants, F-74310 Les Houches +33 () 50544069 / $50555325 \quad$ A: 1 Mar $93 / \mathrm{PP} / 50$ / inv., French / FF $4600 . ;$ incl board, lodge; incl. proc. NATO

July 1- 15 Nonlinear Optical Materials \& Devices for Applicns. in Information Tech.: Int. School of Materials Sci. \& Tech. Erice, Italy Dr. Welford, DRA, RSRE, St. Andrews Rd., Malvern, Worcs. WR14 3 PS, UK +44(684) 895015 / $894540 \quad$ PP / $80 /$ SUS 700.-; incl. board, lodge; incl. proc. NATO 
20-30 Positron Studies of Solids: Int. School of Physics "Enrico Fermi" - 2nd Course Varenna, Italy A. Dupasquier, Dip. di Fisica, Politecnico di Milano piazza L. da Vinci, 32, 1-20133 Milano +39 (2) 23 $996121 / 23996126 \quad$ A: 1 May $93 / P P / 50$ / LIT 1700 000.-; incl. board, lodge SIF

20-30 Nonlinear Optical Materials: Int. School of Physics "Enrico Fermi" - 3rd Course Varenna, Italy V. Degiorgio, Dip. di Elettronica dell'Univ., Via Abbiategrasso, 209, piazza L. da Vinci, 32, 1-27100 Pavia +39 (382) $391280 / 422583 \quad$ A: 1 May $93 / \mathrm{PP} /$ 50 / LIT $1700000 .-;$ incl. board, lodge SIF

- Aug./Sept. Experimental Physics for Non-Physicists Bratislava, Czecho-Slovakia J Cermak Phys. Dept, K 2020, Czech Tech. Univ., Technicka 4 CS-166 07 Prague $6+42$ (2) $3322425 / 3112768$ Aug. 1-21 High-Energy Phenomenology: 42nd Scottish University Summer School in Physics St. Andrews, UK A. Walker, Physics Dept., Univ. of Edinburgh, Edinburgh EH9 3JZ, UK +44 (31) 650 $5237 / 6505273$ a.walker @ ed.ac.uk A: $31 \mathrm{Ma}$ 93 / PP / 100.-; incl. board, lodge; incl. proc. NATO 1-28 Cosmology \& Large-Scale Structure: NATO ASI Les Houches, France J. Zinn-Justin, Service de Physique Théorique,Côte de Chavants, F-74310 Les Houches + +33 () 50544069 / $50555325 \quad$ A: 1 Mar $93 / \mathrm{PP} / 50 /$ inv. I FF 3800.-; incl. board, lodge; incl. proc.

18-28 Frontier Topics in Nuclear, AstroNuclear \& Astro-Particle Physics: 23rd Mazurian Lakes Summer School on Nuclear Physics Piaski, Poland Z. Sujkowski, SINS, PL-05-400 Swierk +48 (22) $798627 / 793481 \quad$ sujkowsk @ plearn 24 - Sept. 2 Information sur les sciences et technologies spatiales: Stage Nancy, France CNES - Délégation à la Communication, 18, avenue Edouard-Belin, F-31055 Toulouse Cédex +33 () $61273131 / 61281327$

30 - Sept. 3 Local Transport Studies in Fusion Plasmas: Int. Workshop Varenna, Italy G. Gorini, ISPP, via Celoria, 16, 1-20133 Milano +39 (2) $2392637 / 2392205$ giuseppe @ imisiam.mi.cnr.it A: 10 Jun 93 / Ab: 10 Jun 93 / PP: 3 Sep 93 / 60 / LIT 500 000.-; incl. proc.

- Sept. 6-17 Unsteady Combustion Praia da Granja, Espinho, Portugal M.V. Heitor, Inst. Sup. Técnico, Seccão de Termodinãmica Aplicada, Dept. de Engenharia Mecãnica, Av. Rovisco Pais + +351 (1) $8473453 / 896156$ A: 28 Feb $93 / \mathrm{PP} / 150 /$ SUS 600.-; incl. board, lodge; incl. proc. NATO 6-17 Geometrical Phases: Course Trieste, Italy ICTP, POB 586, I-34100 Trieste +39 (40) 22401 / $224163 \quad$ / lim.

6-24 Soil Physics: College ICTP, POB 586, I-34100 Trieste +39 (40) 22401 /

Trieste, Italy 7-17 Hochenergiephysik: 25. Herbstschule Maria Laach, Germany H.D. Dahmen, FB 7, Physik, Univ.-GH-Siegen, Adolf-Reichwein-Str., W-5900 Siegen +49(271) $7404132 / 740233070 /$ inv. German only / DM 430.--; incl. board, lodge

8-17 Neutrinos in Cosmology, in Astro, Particle \& Nuclear Physics: Int. School of Nuclear Physics (15th Course) Erice, Italy A. Faessler, Inst. f. Theo. Physik, Univ. of Tübingen, Auf der Morgenstelle 14, W-7400 Tübingen +49 (7071) $296370 / 296400$ faessler @ mailserv.zdv.uni-tuebingen.de A: $15 \mathrm{Jul} 93$ PP / SFR 900.-

13-24 High-Energy Physics: 1993 European Schoo Cracow region, Poland S. Tracy, CERN-DG, $\mathrm{CH}-1211$ Geneva $23 \quad-/+41$ (22) 7823011 tracey @ cernvm.cern.ch A: 20 Jun 93/PP/120/ inv. I=SFR 1500.-; 100.- students; incl. board, lodge; incl. proc.

- Oct. Technologie des véhicules spatiaux Cours de technologie spatiale Toulouse, France CNES - Délégation à la Communication, 18, avenue Edouard-Belin, F-31055 Toulouse Cédex +33 () $61273131 / 61281327$

25 - Nov. 19 Use of Radiation in Sci. \& Technology - John Fuggle Memorial: 2nd School Trieste, Italy ICTP, POB 586, 1-34100 Trieste $\quad+39(40)$ $22401 / 224163$

- Nov.22-26 Chemical Sensors: EUROCOURSE Ispra, Italy Eurocourses, JRC, 1-21020 Ispra +39 (332) $787919 / 789839$

23 - Dec. 5 Adv. Electronic Materials: Int. School Madras, India $\quad$ C. Paorici, Physics Dept., Univ. of Parma, 1-43100 Parma

\section{Programmes 1993/4}

mid-Feb. - Dec. 17 Seattle, WA, USA National Institute for Nuclear Theory

Nuclear Physics in Atoms \& Molecules

mid-Feb. mid-March

Phenomenology \& Lattice QCD mid-June - 3 Sept. Large Amplitude Collective Motion 4 Oct. - 17 Dec. E. Henley, NINT, Univ. of Washington, Seattle, WA 98195, USA

+ 1 (206) 5432896 / - henley @ uwaphast July Cambridge, UK

Isaac Newton Inst. for Mathematical Physics Random Spatial Processes July - Dec. 1993 Geometry \& Gravitation Jan. - June 1994 Cellual Automata, Aggreg. \& Growth Jan. - June '94 P. Goddard, DAMTP, Cambridge Univ., Silver Street, Cambridge CB3 9EW, UK

+ 44 (223) 337883 / - pg1 @ uk.ac.rl.ib
Europhysics Notes (continued from page 186)

- UK Defense Agency to Save 90 M乏

Reduced east-west tension means that the UK's Defense Research Agency (120000 staff), whose main client is the Ministry of Defense, has announced a major reorganization aimed a saving $90 \mathrm{ME}$ in running costs. The DRA was formed out of five separate organizations that evolved in an era of global interests; it is to be reshaped to improve costeffectiveness. Half the savings will come from sharing more services and the remainder from closing smaller sites. So today's 41 manned sites will be trimmed to three major sites (Malvern, Fort Halstead and Farnbo-

\section{UPPSALA UNIVERSITET}

\section{RESEARCH ASSOCIATE IN MOLECULAR LASER SPECTROSCOPY}

The ESCA-LASER laboratory at Uppsala University has equipment for electron, ion and time of flight spectrometry. Several lasers, at present two excimer lasers, one NdYAG laser and two tunable dye lasers (type Lambda Physik) in the nano- and femtosecond range are connected to the spectrometers, built at the ESCA-LASER laboratory. These have a supersonic jet arrangement (nozzle and skimmer) that can be used for research on molecules as well as metal- and molecule clusters with a high resolution. The research group is composed of Swedish and foreign gues researchers.

The ESCA-LASER laboratory wants a research associate in Molecular Laser Spectroscopy to do basic research in this field. The candidate is also expected to take active part in any upgrading of the system.

The applicant should have a doctor's degree, obtained not more than five years ago, and the position will be for two years with a possibility of another two years' continuation. A good knowledge of English is essential.

Information can be obtained from Professor Kai Siegbahn, ESCA-LASER laboratory, Physics Department, Uppsala University, Box 530, S-751 21 Uppsala, Sweden, tel.: +46 (18) 1835 59, fax: +46 (18) 554549 and about salary, etc. from Sigbrit Hermodsson, tel.: +46 (18) 181732.

Please send your complete application, incl. C.V., copies of degree certificates, an account of previous research achievements and teaching merits, a list of publications and reprints according to the list to the Registrar, Uppsala University, Box 256, S-751 05 Uppsala, Sweden. The application should arrive there not later than 30 December 1992.

The forthcoming deadline for applications for magnet time allocation (February to July 1993) at the

\section{GRENOBLE HIGH MAGNETIC FIELD LABORATORY}

is December 15, 1992.

Scientists of EEC countries are entitled to apply under the "Large Installation Plan" programme. Application forms are available on request.

Please contact: $\quad$ G. MARET

Laboratoire des Champs Magnétiques Intenses de Grenoble,

Centre National de la Recherche Scientifique et

Max-Planck-Institut für Festkörperforschung

B.P. 166 - F-38042 Grenoble Cédex 9 / FRANCE

Tel.: +33() 76881001

Fax: $+33($ ) 76872197

Bitnet: MPI20 @ FRGREN81

\section{POST-DOCTORAL POSITIONS - SISSA/ISAS TRIESTE}

The International School for Advanced Studies (SISSA/ISAS) in Trieste expects to offer a number of post-doctoral positions in the following fields:

- Nonlinear Analysis and Geometry

- Mathematical Physics

- Theoretical Particle Physics

These positions will be available from the Fall of 1993 for one year and renewable for a second year. Candidates, who must not be over 36 years of age, should submit their applications by 16 January 1993 with their Curriculum Vitae, list of published works and their research programme. They should arrange for 2 letters of reference to be sent by the same date.

Applications and correspondence should be sent to: Postdoc Programme, International School for Advanced Studies, Via Beirut 2-4, I-34013 TRIESTE, ITALY 